\title{
Checklist of the flora in ironstone outcrops at the Urucum Plateau, Corumbá, Mato Grosso do Sul
}

\author{
Michele Soares de Lima ${ }^{1,3 *}$, Adriana Takahasi², Geraldo Alves Damasceno-Junior ${ }^{3}$ \\ \& Andréa Cardoso Araujo ${ }^{3}$ \\ ${ }^{1}$ Instituto Federal de Educação, Ciência e Tecnologia de Mato Grosso do Sul, Laboratório de Biologia, Campus \\ Corumbá, R. Pedro de Medeiros, 79310-110, Corumbá, MS, Brasil \\ ${ }^{2}$ Universidade Federal de Mato Grosso do Sul, Faculdade de Engenharias, Arquitetura e Urbanismo e \\ Geografia, Cidade Universitária, 79070-900, Campo Grande, MS, Brasil \\ ${ }^{3}$ Universidade Federal de Mato Grosso do Sul, Laboratório de Ecologia, Instituto de Biociências, Cidade \\ Universitária, 79070-900, Campo Grande, MS, Brasil \\ *Corresponding author: Michele Soares de Lima, e-mail: michele.lima@ifms.edu.br
}

LIMA, M.S., TAKAHASI, A., DAMASCENO-JUNIOR, G.A., ARAUJO, A.C. Checklist of the flora in ironstone outcrops at the Urucum Plateau, Corumbá, Mato Grosso do Sul. Biota Neotropica. 19(3): e20180708. http://dx.doi.org/10.1590/1676-0611-BN-2018-0708

\begin{abstract}
Cangas" are ironstone outcrops occurring at the foot of the Urucum Plateau, on the western edge of Pantanal, Corumbá-MS. In Brazil, the knowledge about flora and ecology of the plant communities associated with these formations is still incipient. These habitats are among the most threatened and less studied in Brazil because of their association with high quality iron ore deposits. We present a cheklist of the flora from these formations, resulting from different authors' collecting efforts in 10 areas of the Plateau. A total of 302 species have been recorded; they were distributed in 53 botanical families and 175 genera. Poaceae (43 species), Fabaceae (41), Euphorbiaceae (22), Cyperaceae (19), Malvaceae (19), Convolvulaceae (15), Malpighiaceae (13) and Apocynaceae (11) accounted for $60.6 \%$ of the species richness. Our data add 59 new occurrences of species of angiosperms to the ironstone outcrops flora in the Urucum Plateau. Among these, 27 species had no occurrence record for Mato Grosso do Sul and three species had not yet been recorded for Brazil. The high diversity and presence of endemic, rare, endangered and/or not yet cataloged species for the Urucum Plateau region, reinforces the need for local conservation units that can guarantee the preservation of these species, since the existing environmental protection areas are insufficient to guarantee the maintenance of typical species from this habitat in the region.
\end{abstract}

Keywords: biodiversity, ferruginous geosystem, Pantanal.

\section{Checklist da flora de cangas do Maciço do Urucum, Corumbá, Mato Grosso do Sul}

Resumo: As cangas são afloramentos ferruginosos que ocorrem ao sopé do Maciço do Urucum, na Borda Oeste do Pantanal, Corumbá-MS. No Brasil, o conhecimento sobre a florística e ecologia das comunidades vegetais associadas a essas formações ainda é incipiente. Esses habitats estão entre os mais ameaçados e menos estudados do Brasil, devido à sua associação a depósitos de minério de ferro de alta qualidade. Nós apresentamos um cheklist da flora desses ambientes, resultante de diversos esforços de coleta de diferentes autores em 10 áreas do Maciço. Foram registradas 302 espécies distribuídas em 53 famílias botânicas e 175 gêneros. Poaceae (43 espécies), Fabaceae (41), Euphorbiaceae (22), Cyperaceae (19), Malvaceae (19), Convolvulaceae (15), Malpighiaceae (13) e Apocynaceae (11) representaram 60,6\% da riqueza específica. Nossos dados adicionam 59 novas ocorrências de espécies de angiospermas para a flora de cangas do Maciço do Urucum. Das espécies apresentadas, 27 ainda não possuíam registro de ocorrência para o Mato Grosso do Sul e três espécies não haviam sido ainda registradas para o Brasil. A alta diversidade e a presença de espécies endêmicas, raras, ameaçadas e/ou ainda não catalogadas para a região do Maciço do Urucum reforça a necessidade de unidades de conservação locais que possam garantir a preservação dessas espécies, uma vez que as áreas de proteção ambiental existentes na região são insuficientes para garantir a manutenção de populações típicas desse habitat.

Palavras-chave: biodiversidade, geossistema ferruginoso, Pantanal. 


\section{Introduction}

Ferruginous geosystems are landscapes of great value due to the uniqueness of their flora and fauna, presence of endemic species and/ or provided ecosystem services (Tibbett 2015). Due to the soil porosity and permeability in these formations, they have high water recharge capacity and storage, forming large aquifers that supply springs and cities (Carmo et al. 2012). However, because they are located in regions with large mineral deposits of economic interest, they are among the most endangered landscapes in the world (Jacobi \& Carmo 2008a, Tibbett 2015).

These environments, originated in the Archean and Paleoproterozoic period, are usually located in mountain tops, but can also be found in foothills (Souza \& Carmo 2015). In addition to presenting high biological value, these environments harbor sites of inestimable archaeological value and important water resource deposits (Jacobi et al. 2015, Souza \& Carmo 2015). In Brazil, ferruginous geosystems occur in the states of Bahia, Minas Gerais, Mato Grosso do Sul and Pará, and they are important mineral exploration areas (Carmo et al. 2012). These geosystems, known in Brazil as "cangas", usually occur in higher relief areas, with tabular tops (Souza \& Carmo 2015). However, in the Urucum Plateau region, located in the municipalities of Corumbá and Ladário, state of Mato Grosso do Sul, they not only occur on tops but also in low altimetric levels and slopes (Del'arco et al. 1982, Takahasi 2015). These low altimetric ironstone outcrops are called "bancadas lateríticas" (Takahasi \& Meirelles 2014).

The Urucum Plateau represents the lithostratigraphic unit of most economic interest in Mato Grosso do Sul, due to the presence of important deposits of iron and manganese (Del'arco et al. 1982). Ironstone outcrops of Urucum plateau are Quaternary deposit formations produced under climatic conditions different from those of the current era. They probably occurred at the time of the Paraguay River Depression and the plio-plestocene plediplain origin, period in which a semi-arid weather subjected to torrential rains and erosive processes prevailed (Del'arco et al. 1982).

In Brazil, the knowledge about floristic and ecology of plant communities associated with ferruginous geosystems is still incipient (Jacobi \& Carmo 2011, Jacobi et al. 2015). These habitats are among the most threatened and least studied in Brazil respectively due to their association with iron ore deposits with high quality and the difficulty of access (Jacobi \& Carmo 2008a). Studies on ironstone outcrops flora are recent and concentrated largely on the Iron Quadrangle, in the state of Minas Gerais (Mourão \& Stehmann 2007, Viana \& Lombardi 2007, Pifano et al. 2010, Ataíde et al. 2011, Carmo \& Jacobi 2013). The results obtained by those studies indicate that ironstone outcrops harbor a large number of plant species, contributing to the increase of the alpha diversity of the regions where they occur (Jacobi \& Carmo 2008a).

In the region of Corumbá, some ironstone outcrops in the Urucum plateau have been studied for their ecological aspects (Takahasi 2010, Takahasi \& Meirelles 2014, Oliveira 2016) and flora (Takahasi 2010, Takahasi 2015); but there is no listing that includes all species already collected in the region. In this study we present a checklist of species of Angiosperms from the ironstone outcrops in Urucum Plateau, based on several collecting efforts carried out in region from the 1990s to the present time, in order to fill the information gaps about the flora of these environments and evaluate the similarity between the ferruginous outcrops from Mato Grosso do Sul and those ferruginous outcrops from other regions.

\section{Material and Methods}

The studied ironstone outcrops are located at the foot of the Urucum Plateau Residual, a non-floodable region around the municipalities of Corumbá and Ladário (Figure 1), the western border of Pantanal, Mato Grosso do Sul, Midwest region of Brazil. The plateau, also known as Urucum Massif, is a complex of hills called locally as Ururum, Santa Cruz, Grande, Rabichão, São Domingos and Tromba dos Macacos, whose altitudes vary between 80 to $1.065 \mathrm{~m}$, being the highest hill recognized as the highest point of the State (Borges et al. 1997, Damasceno-Junior 2005). The area covers approximately $1.211 \mathrm{~km}^{2}$ and is bordered on north by the Paraguay River, on west by the Bolivian border, on south and east by the Pantanal floodplain (Silva et al. 2000). Ironstone outcrops from this region occur along the drainage lines at the foot of the plateau hills (about 100 to $150 \mathrm{~m}$ altitude), and due to the predominantly flat terrain, small pools of water may accumulate during the rainy season.

Ten ironstone outcrops were included in this inventory: Pantanal Park Road, Band'alta farm, Monjolinho farm, Figueira farm, São João farm, São Sebastião do Carandá farm, two sites in the Municipal Natural Park Piraputangas, Uruba farm and Rabicho farm (Table 1).

In the Urucum Plateau region, the average annual temperature is $25.1^{\circ} \mathrm{C}$ and the average rainfall is $1.070 \mathrm{~mm}$ annually (Soriano 1997). There are two well-defined seasons, a dry season that runs from May to September and a rainy season from October to March, with $45 \%$ of rainfall occurring from December to February (Loureiro et al. 1982).

The vegetation surrounding these ironstone outcrops is a Submontane Seasonal Decidual Forest (Damasceno-Junior 2005). We can characterize three habitats in ironstone outcrops at Urucum Plateau: the first one is hardened ferruginous substrate where plants established directly on them or in places with a thin layer of sediment. The second one consists of soil islands, specially mats of monocotyledons, with a deeper layer of soil that allows establishment of phanerophytes. Finally, the third one is constituted by ephemeral flush communities that occurs on runoff-habitats like the slopes of ironstone outcrops allowing the establishment of typical flooded plant species.

The checklist of the ironstone outcrops flora in the Urucum Plateau was elaborated through data collected in Fazenda Band'alta, from January 2017 to July 2018; in researches made by the authors over several years in the outcrops from the region and Herbaria queries at Universidade Federal de Mato Grosso do Sul, Campus Corumbá (COR) and Campo Grande (CGMS); Herbarium of Embrapa Pantanal (CPAP); Herbarium Friburguense of Pontifícia Universidade Católica do Rio de Janeiro (FCAB); Herbarium of Universidade Estadual de Campinas (UEC); Herbarium of Instituto de Biociências at Universidade do Rio Grande do Sul (ICN); Herbarium Maria Eneyda P. K. Fidalgo, at Instituto de Botânica de São Paulo (SP) and Herbarium of Universidade de Brasília (UB). The obtained dataset covers a large part of the ironstone outcrops flora in the region, and was later supplemented with herbaria data available on internet (CRIA 2018, Flora do Brasil 2020, 

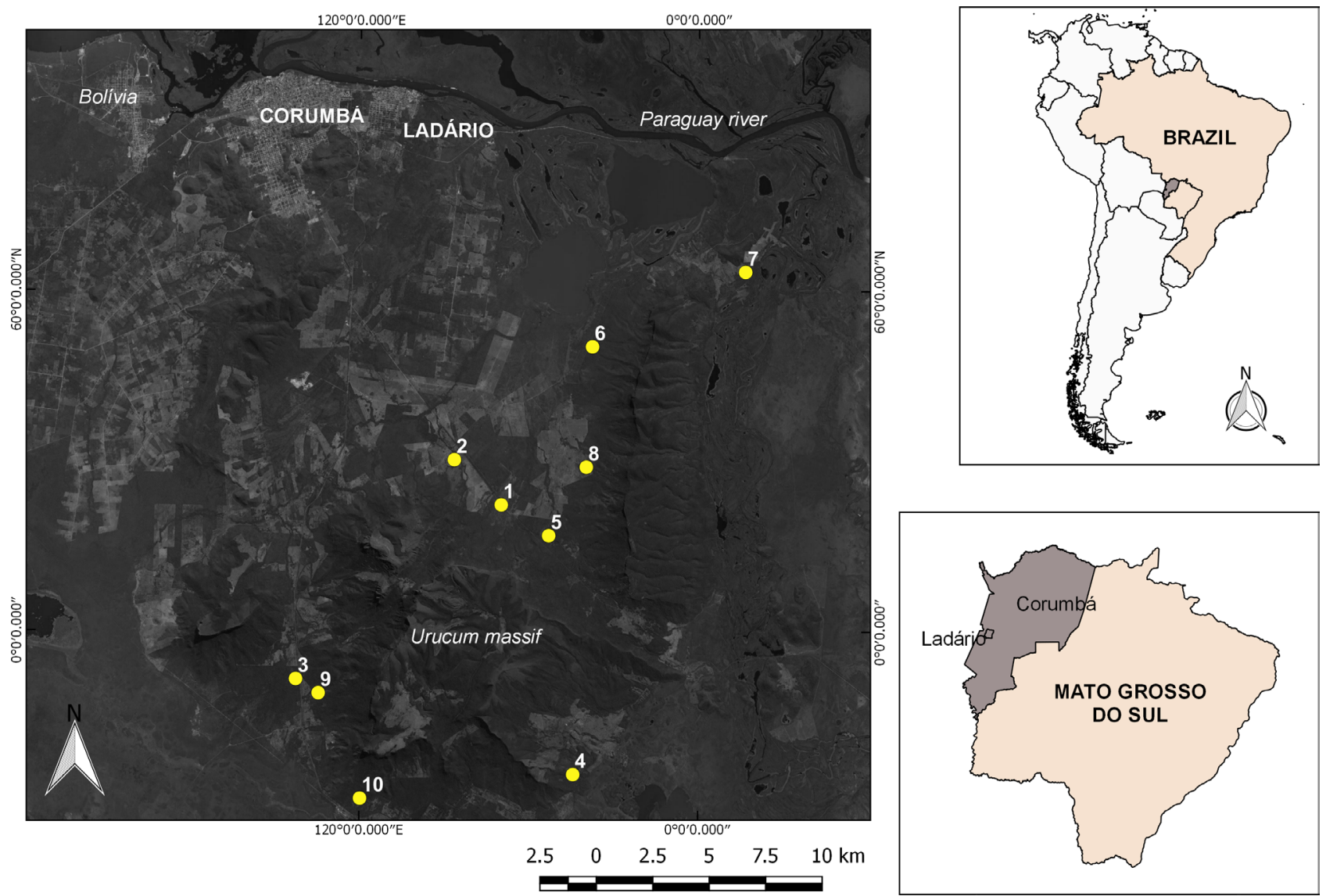

Figure 1. Location of the ten analyzed cangas (yellow dots) at the Urucum Plateau, Western Border of Pantanal, MS.

Table 1. Locations included in this study with their respective geographical coordinates.

\begin{tabular}{|c|c|c|}
\hline $\begin{array}{l}\text { Sampling } \\
\text { points }\end{array}$ & Locality & $\begin{array}{c}\text { Geographical } \\
\text { coordinates }\end{array}$ \\
\hline 1 & Pantanal Park Road & $\begin{array}{l}19^{\circ} 10^{\prime} 02.0^{\prime \prime} \mathrm{S}, \\
57^{\circ} 33^{\prime} 31.0^{\prime \prime} \mathrm{W}\end{array}$ \\
\hline 2 & Band'Alta farm & $\begin{array}{l}19^{\circ} 08^{\prime} 57.3^{\prime \prime} \mathrm{S} \\
57^{\circ} 34^{\prime} 42.1^{\prime \prime} \mathrm{W}\end{array}$ \\
\hline 3 & Figueira farm & $\begin{array}{l}19^{\circ} 14^{\prime} 10.7^{\prime \prime} \mathrm{S}, \\
57^{\circ} 38^{\prime} 43.1^{\prime \prime} \mathrm{W}\end{array}$ \\
\hline 4 & Monjolinho farm & $\begin{array}{l}19^{\circ} 16^{\prime} 28.4^{\prime \prime S} \\
57^{\circ} 31^{\prime} 42.7^{\prime \prime} \mathrm{W}\end{array}$ \\
\hline 5 & São João farm & $\begin{array}{l}19^{\circ} 10^{\prime} 46.2^{\prime \prime} \mathrm{S} \\
57^{\circ} 32^{\prime} 19.1^{\prime \prime} \mathrm{W}\end{array}$ \\
\hline 6 & São Sebastião do Carandá farm & $\begin{array}{l}19^{\circ} 06^{\prime} 15.5 ” \mathrm{~S}, \\
57^{\circ} 31^{\prime} 12.5^{\prime \prime} \mathrm{W}\end{array}$ \\
\hline 7 & Uruba farm & $\begin{array}{l}19^{\circ} 04^{\prime} 28.8^{\prime \prime} \mathrm{S} \\
57^{\circ} 27^{\prime} 20.2^{\prime \prime} \mathrm{W}\end{array}$ \\
\hline 8 & Rabicho farm & $\begin{array}{l}19^{\circ} 09^{\prime} 08.0 " \mathrm{~S}, \\
57^{\circ} 31^{\prime} 22.0^{\prime \prime} \mathrm{W}\end{array}$ \\
\hline 9 & $\begin{array}{c}\text { Municipal Natural Park } \\
\text { Piraputangas }\end{array}$ & $\begin{array}{l}19^{\circ} 14^{\prime} 31.0^{\prime \prime} \mathrm{S}, \\
57^{\circ} 38^{\prime} 08.0^{\prime \prime} \mathrm{W}\end{array}$ \\
\hline 10 & $\begin{array}{c}\text { Municipal Natural Park } \\
\text { Piraputangas }\end{array}$ & $\begin{array}{l}19^{\circ} 17^{\prime} 02.0^{\prime \prime} \mathrm{S}, \\
57^{\circ} 37^{\prime} 06.0^{\prime \prime} \mathrm{W}\end{array}$ \\
\hline
\end{tabular}

Jabot 2018). We considered only specimens identified at the taxonomic level of species and excluded those with dubious identification or at the genera-level. The taxa classification followed the APG IV system (2016), and the nomenclature and synonymizations were updated according to Flora do Brasil 2020 (2018). The similarity in species composition among the 10 analyzed ironstone outcrops was calculated using the Sorensen Similarity Index.

\section{Results}

A total of 302 species distributed in 53 botanical families and 175 genera were cataloged (Table 2). The richest species families were Poaceae (43 species), Fabaceae (41), Euphorbiaceae (22), Malvaceae (19), Cyperaceae (19), Convolvulaceae (15), Malpighiaceae (13) and Apocynaceae (11) that represented $60.6 \%$ of the specific richness found in the ironstone outcropsof the region (Figure 2). Poaceae, Fabaceae, Euphorbiaceae and Malvaceae are also the families with the highest number of genera, being Cyperus (9), Croton (8), Mimosa (7), Portulaca (7) and Evolvulus (6) the most common genera.

In the soil islands, there are deciduous forest species as Aspidosperma quirandy Hassl., Pseudobombax marginatum (A.St.Hil.) A.Robyns and Myracrodruon urundeuva Allemão, whereas in areas where there is a thin layer of soil only herbaceous species can be established, in many cases annuals for example, Gomphrena centrota 
Lima, M.S. et al.

Table 2. List of Angiosperms species from the cangas at the Urucum Plateau, Western Border of Pantanal, MS, with respective growth habits, Vouchers (collector's name, collection number and registration in the herbarium where the specimen is deposited, when possible ), place of occurrence and state of conservation of the species according to criteria of the International Union of Conservation and Natural Resources (IUCN 2018) and Livro Vermelho da Flora do Brasil pela CNCFlora (Martinelli \& Moraes 2013): $\mathrm{CR}=$ critically endangered, $\mathrm{EN}=$ endangered, $\mathrm{LC}=$ least concern, $\mathrm{NT}=$ near threatened, $\mathrm{VU}=$ vulnerable, $\mathrm{DD}=$ data deficient, "$=$ not evaluted. The numbers of localities are shown in Table 1 .

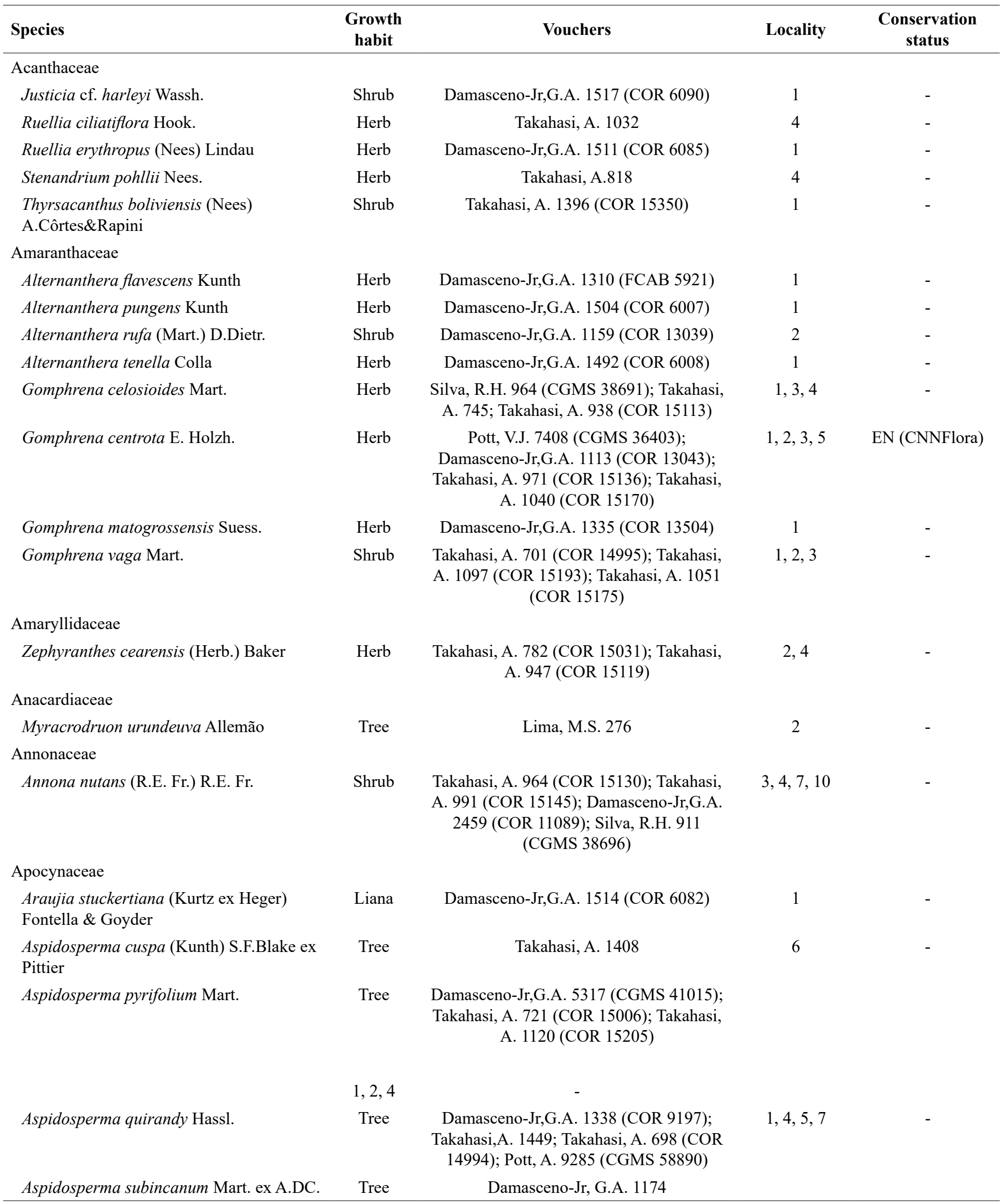


Continuation Table 2.

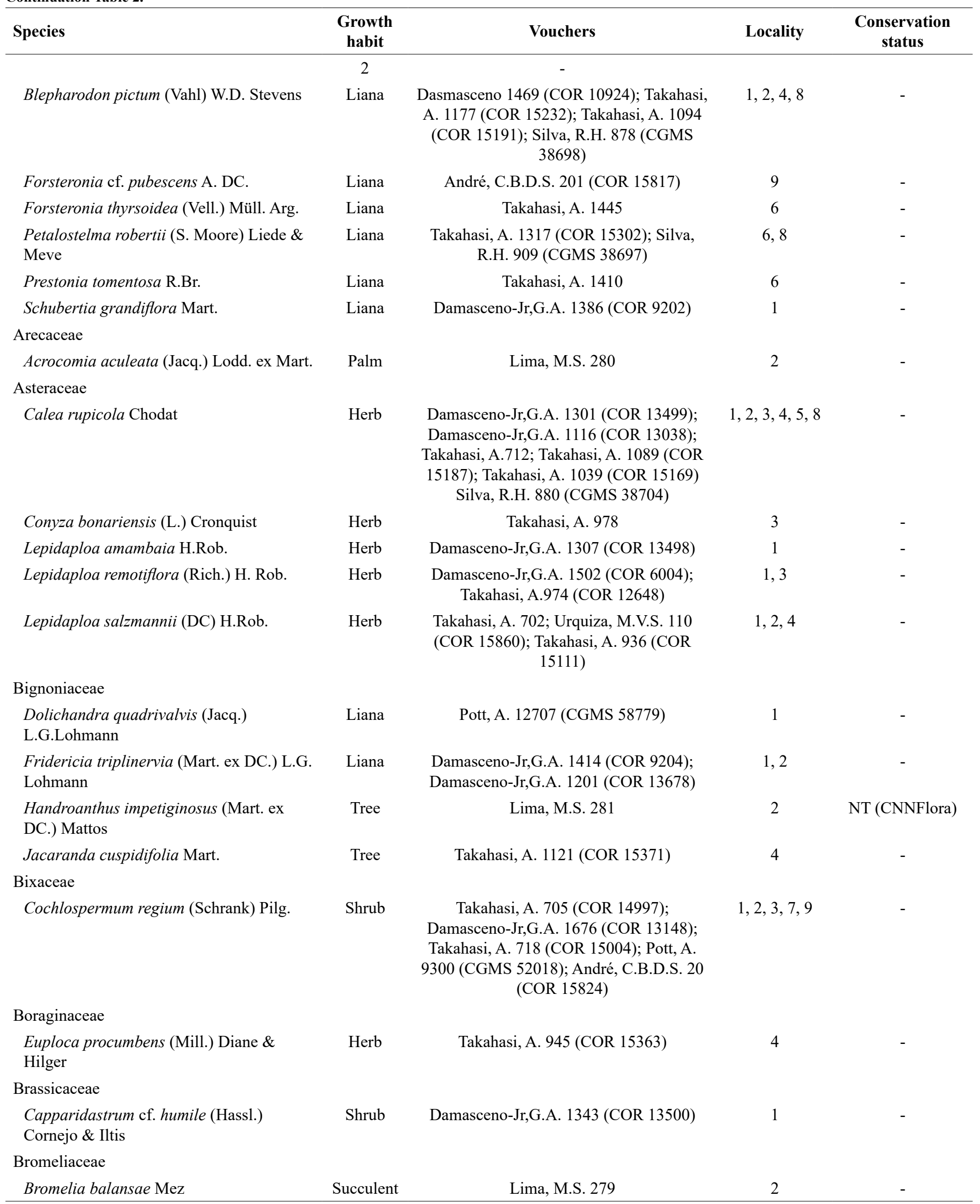


Lima, M.S. et al.

Continuation Table 2.

\begin{tabular}{|c|c|c|c|c|}
\hline Species & $\begin{array}{c}\text { Growth } \\
\text { habit }\end{array}$ & Vouchers & Locality & $\begin{array}{l}\text { Conservation } \\
\text { status }\end{array}$ \\
\hline Deuterocohnia meziana Kuntze ex Mez & Succulent & $\begin{array}{c}\text { Damasceno-Jr, G.A. } 5316 \text { (CGMS 41014); } \\
\text { Takahasi, A. } 722 \text { (COR 15007); Takahasi, } \\
\text { A. } 751\end{array}$ & $1,2,3$ & VU (CNNFlora) \\
\hline Dyckia aff. gracilis $\mathrm{Mez}$ & Succulent & $\begin{array}{c}\text { Damasceno-Jr,G.A. } 1404 \text { (COR 10917); } \\
\text { Ishii, I.H. } 743 \text { (COR 13576) }\end{array}$ & 1,5 & - \\
\hline Dyckia leptostachya Baker & Succulent & $\begin{array}{c}\text { Ishii } 746 \text { (COR 15872); Ishii, I.H. } 747 \\
\text { (COR 15389); Ishii, I.H.786 (COR 15730) }\end{array}$ & $1,2,5$ & - \\
\hline $\begin{array}{l}\text { Tillandsia loliacea Mart. ex Schult \& } \\
\text { Schult f. }\end{array}$ & Epiphyte & Takahasi, A. 1091 & 4 & - \\
\hline \multicolumn{5}{|l|}{ Burseraceae } \\
\hline Cereus bicolor Rizzini \& A.Mattos & Succulent & Takahasi, A. 1376 (COR 15342) & 4 & LC (IUCN) \\
\hline Discocactus ferricola Buining \& Brederoo & $\begin{array}{c}\text { Succulent } \\
1,2,3\end{array}$ & $\begin{array}{l}\text { Garcia, J.S. } 55 \text { (CGMS17537), } \\
\text { EN (IUCN), DD (CNNFlora) }\end{array}$ & & \\
\hline $\begin{array}{l}\text { Frailea cataphracta (Dams) Britton \& } \\
\text { Rose }\end{array}$ & Succulent & Takahasi, A. 779 (COR 15028) & 2 & NT (IUCN) \\
\hline $\begin{array}{l}\text { Harrisia balansae (K. Schum.) N.P. Taylor } \\
\text { \& Zappi }\end{array}$ & Succulent & Takahasi, A. 755 (COR 15017) & 2 & LC (IUCN) \\
\hline Opuntia retrorsa Speg. & Succulent & $\begin{array}{l}\text { Takahasi, A. } 672 \text { (COR 14895); Lima, M.S. } \\
282\end{array}$ & 1,2 & - \\
\hline \multicolumn{5}{|l|}{ Cannabaceae } \\
\hline \multicolumn{5}{|l|}{ Caryophyllaceae } \\
\hline Polycarpaea corymbosa (L.) Lam. & Herb & $\begin{array}{l}\text { Takahasi, A. } 680 \text { (COR 14990); Takahasi, } \\
\text { A. } 973 \text { (COR 15138); Takahasi, A.875; } \\
\text { Silva, R.H. } 899 \text { (CGMS 38728) }\end{array}$ & $1,3,5,8$ & - \\
\hline \multicolumn{5}{|l|}{ Cleomaceae } \\
\hline Physostemon guianense (Aubl.) Malme & Herb & $\begin{array}{c}\text { Damasceno-Jr,G.A. } 1434 \text { (COR 13866); } \\
\text { Damasceno-Jr,G.A. } 1677 \text { (COR 13676); } \\
\text { Takahasi, A. } 815 \text { (COR 15051); Pott, A. } \\
\text { 4784 (CPAP 25125) }\end{array}$ & $1,2,4,7$ & - \\
\hline $\begin{array}{l}\text { Tarenaya eosina (J.F.Macbr.) Soares Neto } \\
\text { \& Roalson }\end{array}$ & Herb & $\begin{array}{c}\text { Damasceno-Jr,G.A. } 1312 \text { (COR 13865); } \\
\text { Takahasi, A. } 1002 \text { (COR 15150); Takahasi, } \\
\text { A. } 915 \text { (COR 15100); Silva, R.H. } 882 \\
\text { (CGMS 38729) }\end{array}$ & $1,2,3,8$ & - \\
\hline \multicolumn{5}{|l|}{ Combretaceae } \\
\hline Combretum duarteanum Cambess. & Shrub & $\begin{array}{c}\text { Damasceno-Jr,G.A. } 1304 \text { (COR 13864); } \\
\text { Damasceno-Jr,G.A. } 1155 \text { (COR 13618); } \\
\text { Takahasi, A. } 825 \text { (COR 15059) }\end{array}$ & $1,2,4$ & - \\
\hline Combretum leprosum Mart. & Tree & Takahasi, A. 766 (COR 15021) & 2 & - \\
\hline Combretum mellifluum Eichler & Tree & Pott, A. 9271 (CPAP 22949) & 7 & - \\
\hline
\end{tabular}


Continuation Table 2.




Lima, M.S. et al.

Continuation Table 2.

\begin{tabular}{|c|c|c|c|c|}
\hline Species & $\begin{array}{c}\text { Growth } \\
\text { habit }\end{array}$ & Vouchers & Locality & $\begin{array}{c}\text { Conservation } \\
\text { status }\end{array}$ \\
\hline Cyperus flavescens L. & Herb & Silva, R.H. 940 (CGMS 38751) & 9 & - \\
\hline Cyperus subcastaneus D.A. Simpson & Herb & Takahasi, A. 1379 (COR 12565) & 6 & - \\
\hline Cyperus surinamensis Rottb. & Herb & $\begin{array}{c}\text { Takahasi, A. } 868 \text { (COR 15075); Takahasi, } \\
\text { A. } 793 \text { (COR 12560) }\end{array}$ & 2,3 & - \\
\hline Cyperus uncinulatus Schrad. ex Nees & Herb & $\begin{array}{c}\text { Takahasi, A. } 1129 \text { (COR 12547); Takahasi, } \\
\text { A. } 835 \text { (COR 15064); Takahasi, A. } 880 \\
\text { (COR 15082) }\end{array}$ & $2,4,5$ & - \\
\hline Eleocharis contracta Maury ex Micheli & Herb & Damasceno-Jr,G.A. 2412 (COR 11069) & 1 & - \\
\hline Eleocharis nigrescens (Nees) Kunth & Herb & Takahasi, A. 1239 (COR 12559) & 2 & - \\
\hline Fimbristylis miliacea (L.) Vahl & Herb & Damasceno-Jr, G.A. 2413A & 1 & - \\
\hline Kyllinga odorata Vahl & Herb & $\begin{array}{c}\text { Takahasi, A. } 1182 \text { (COR 15234); Takahasi, } \\
\text { A. } 1268 \text { (ICN 156813) }\end{array}$ & 2,4 & - \\
\hline Rhynchospora contracta (Nees) J.Raynal & Herb & $\begin{array}{l}\text { Damasceno-Jr, G.A. } 2413 \text { (COR 15913); } \\
\text { Takahasi, A. } 1228 \text { (COR 15256) }\end{array}$ & 1,2 & - \\
\hline \multicolumn{5}{|l|}{ Dioscoreaceae } \\
\hline Dioscorea acanthogene Rusby & Liana & Damasceno-Jr, G.A. 2880 (11122) & 6 & - \\
\hline Dioscorea trifida L.f. & Liana & Silva, R.H. 944 (CGMS 38753) & 4 & - \\
\hline \multicolumn{5}{|l|}{ Erythroxylaceae } \\
\hline Acalypha villosa Jacq. & Shrub & Takahasi, A. 949 (COR 12389) & 4 & - \\
\hline Actinostemon klotzschii (Didr.) Pax & Shrub & $\begin{array}{c}\text { Takahasi, A. } 741 \text { (12395); Takahasi, A. } \\
1119 \text { COR 12392) }\end{array}$ & 3,6 & - \\
\hline Astraea lobata (L.) Klotzch & Herb & Takahasi, A. 832 COR 12384) & 3 & - \\
\hline Cnidoscolus urens (L.) Arthur & Shrub & Takahasi, A. 1304 (COR 15291) & 2 & - \\
\hline $\begin{array}{l}\text { Cnidoscolus vitifolius var. cnicodendrum } \\
\text { (Griseb.) Lourteig \& O'Donnell }\end{array}$ & Tree & Damasceno-Jr,G.A. 1397 (COR 13885) & 1 & - \\
\hline Croton antisyphiliticus Mart. & Shrub & Takahasi, A. 929 (15107) & 3 & - \\
\hline Croton campestris A. St. Hill & Shrub & André, C.B.D.S 12 (COR 15836) & 9 & - \\
\hline Croton corumbensis S.Moore & Shrub & Takahasi, A. 801 & 9 & - \\
\hline Croton didrichsenii G.L.Webster & Shrub & Takahasi, A. 801 (COR 12382) & 3 & - \\
\hline Croton glandulosus $\mathrm{L}$. & Herb & Takahasi, A. 1320 (COR 15304) & 2 & - \\
\hline Croton pedicellatus Kunth & Herb & Takahasi, A. 1246 (COR 15264) & 4 & - \\
\hline Croton sarcopetaloides S.Moore & Shrub & Silva, R.H. 931 (CGMS 38773) & 9 & - \\
\hline Croton triqueter Lam. & Shrub & Takahasi, A. 892 (COR 12385) & 3 & - \\
\hline Dalechampia brasiliensis Lam. & Liana & Takahasi, A. 1247 (COR 15265) & 4 & - \\
\hline Euphorbia thymifolia L. & Herb & Takahasi,A. 1370 (COR 15336) & 4 & - \\
\hline
\end{tabular}


Continuation Table 2.

\begin{tabular}{|c|c|c|c|c|}
\hline Species & $\begin{array}{c}\text { Growth } \\
\text { habit }\end{array}$ & Vouchers & Locality & $\begin{array}{c}\text { Conservation } \\
\text { status }\end{array}$ \\
\hline Jatropha ribifolia (Pohl) Baill. & Shrub & $\begin{array}{l}\text { Takahasi, A. } 706 \text { (COR 14998); Takahasi, } \\
\text { A. 759(COR 15018); Takahasi, A. } 710 \\
\text { (COR 15001) }\end{array}$ & $1,2,3$ & - \\
\hline Jatropha weddeliana Baill. & Shrub & Damasceno-Jr,G.A. Jr 1316 (COR 13883) & 1 & - \\
\hline Manihot anomala Pohl & Shrub & $\begin{array}{c}\text { Takahasi, A. } 806 \text { (COR 12383); Silva, R.H. } \\
919 \text { (CGMS 38771) }\end{array}$ & 3,10 & - \\
\hline Manihot guaranitica Chodat \& Hassl. & Shrub & Takahasi, A. 767 (COR 15022) & 2 & - \\
\hline Microstachys hispida (Mart.) Govaerts & Herb & $\begin{array}{c}\text { Takahasi, A. } 1028 \text { (COR 15165); Silva, } \\
\text { R.H. } 937 \text { (CGMS 38774) }\end{array}$ & 3,9 & - \\
\hline \multicolumn{5}{|l|}{ Fabaceae } \\
\hline Aeschynomene histrix Poir. & Shrub & $\begin{array}{c}\text { Damasceno-Jr,G.A. 1318; Takahasi, A. } \\
997 \text { (COR 15148); Takahasi, A. } 821 \text { (COR } \\
\text { 15057); Takahasi, A. } 955 \text { (COR 15123); } \\
\text { Pott, V.J. } 4781 \text { (CGMS 53934); Silva, R.H. } \\
877 \text { (CGMS 38777) }\end{array}$ & $1,3,4,6,7,8$ & - \\
\hline Alysicarpus vaginalis (L.) DC. & Herb & Takahasi, A. 1019 (COR 15159) & 2 & - \\
\hline Amburana cearensis (Allemão) A.C. Sm. & Tree & $\begin{array}{l}\text { Lima, M.S. 283; Damasceno-Jr, G.A. 2882; } \\
\text { Silva, R.R } 864 \text { (COR 14763) }\end{array}$ & $2,6,7$ & $\begin{array}{l}\text { EN (IUCN), NT } \\
\quad \text { (CNNFlora) }\end{array}$ \\
\hline Anadenanthera colubrina (Vell.) Brenan & Tree & Lima, M.S. 277 & 2 & - \\
\hline Bauhinia leptantha Malme & Shrub & Silva, R.R 462 (UEC 141018) & 7 & VU (CNNFlora) \\
\hline $\begin{array}{l}\text { Bauhinia pentandra (Bong.) Vogel ex } \\
\text { Steud. }\end{array}$ & Shrub & $\begin{array}{l}\text { Damasceno-Jr,G.A. } 1303 \text { (COR 11173); } \\
\text { Takahasi, A. } 961 \text { (COR 15128); Silva, } \\
\text { R.R. } 634 \text { (UEC 141026); Silva, R.H. } 913 \\
\text { (CGMS 38796) }\end{array}$ & $1,3,7,10$ & - \\
\hline Camptosema ellipticum (Desv.) Burkart & Liana & $\begin{array}{l}\text { Takahasi, A. } 1041 \text { (COR 15171); Takahasi, } \\
\text { A. } 1093 \text { (COR 15190); Takahasi, A. } 1435\end{array}$ & $2,4,6$ & - \\
\hline Chamaecrista nictitans (L.) Moench & Shrub & $\begin{array}{c}\text { Takahasi, A. } 960 \text { (COR 15127); André, } \\
\text { C.B.D.S. } 81 \text { (COR 15840) }\end{array}$ & 3,9 & LC (IUCN) \\
\hline Chamaecrista serpens (L.)Greene & Shrub & $\begin{array}{l}\text { Damasceno-Jr,G.A. } 1302 \text { (COR 12616); } \\
\text { Takahasi, A. } 1006 \text { (COR 15153); Silva, } \\
\text { R.R 546 (COR 14633); Silva, R.H. } 930 \\
\text { (CGMS 38798) }\end{array}$ & $1,2,7,9$ & - \\
\hline $\begin{array}{l}\text { Chamaecrista supplex (Benth.) Britton \& } \\
\text { Killip }\end{array}$ & Herb & Takahasi, A. 885 (COR 15084) & 5 & - \\
\hline Crotalaria pallida Aiton & Shrub & Damasceno-Jr,G.A. 2409 (COR 6462) & 1 & - \\
\hline Dipteryx alata Vogel & Tree & $\begin{array}{c}\text { Takahasi, A. } 1319 \text { (COR 15303); Takahasi, } \\
\text { A. } 684 \text { (COR 14992); Almeida, L.W. } 18 \\
\text { (COR 16107) }\end{array}$ & $2,5,9$ & VU (IUCN) \\
\hline $\begin{array}{l}\text { Guibourtia hymenaefolia (Moric.) J. } \\
\text { Léonard }\end{array}$ & Tree & $\begin{array}{l}\text { Damasceno-Jr,G.A. } 1340 \text { (COR13881); } \\
\text { Takahasi, A. } 1314 \text { (COR 15299) }\end{array}$ & 1,6 & - \\
\hline Hymenaea stigonocarpa Hayne & Tree & Almeida, L.W. 17 (COR 16106) & 9 & - \\
\hline $\begin{array}{l}\text { Macroptilium bracteatum (Nees \& } \\
\text { C.Mart.) Marechal \& Bau }\end{array}$ & Shrub & Takahasi, A. 996 (COR 15147) & 3 & LC (IUCN) \\
\hline Macroptilium lathyroides (L.) Urb. & Liana & Takahasi, A. 1372 (COR 15338) & 2 & - \\
\hline
\end{tabular}


Lima, M.S. et al.

Continuation Table 2.

\begin{tabular}{|c|c|c|c|c|}
\hline Species & $\begin{array}{c}\text { Growth } \\
\text { habit }\end{array}$ & Vouchers & Locality & $\begin{array}{c}\text { Conservation } \\
\text { status }\end{array}$ \\
\hline Mimosa bimucronata (DC.) Kuntze & Shrub & $\begin{array}{c}\text { Silva, R.H. } 965 \text { (CGMS 38784); Takahasi, } \\
\text { A. } 1009 \text { (COR 15154) }\end{array}$ & 1,2 & LC (IUCN) \\
\hline Mimosa candollei R.Grether & Shrub & Silva, R.R 339 (COR 14654) & 7 & - \\
\hline $\begin{array}{l}\text { Mimosa craspedisetosa Fortunato \& } \\
\text { Palese }\end{array}$ & Shrub & Silva, R.R 750 (UEC 143340) & 7 & - \\
\hline Mimosa polycarpa Kunth & Shrub & Silva, R.R. 874 (UEC 142402) & 7 & - \\
\hline $\begin{array}{l}\text { Mimosa sensibilis var. urucumensis } \\
\text { Barneby }\end{array}$ & Shrub & $\begin{array}{c}\text { Takahasi, A. } 1080 \text { (COR 15179); Takahasi, } \\
\text { A. } 1087 \text { (COR 15186); Silva, R.R } 1096 \\
\text { (COR 14687) }\end{array}$ & $3,4,7$ & - \\
\hline $\begin{array}{l}\text { Muellera variabilis (RR.Silva \& AMG. } \\
\text { Azevedo) MJ.Silva \& AMG.Azevedo }\end{array}$ & Tree & $\begin{array}{c}\text { Takahasi, A. } 726 \text { (COR 15010); Takahasi, } \\
\text { A. } 1137 \text { (COR 15215) }\end{array}$ & 2,3 & - \\
\hline $\begin{array}{l}\text { Senegalia cf. martii (Benth.) Seigler \& } \\
\text { Ebinger }\end{array}$ & Shrub & Damasceno-Jr,G.A. 1342 (COR 13709) & 1 & - \\
\hline Senegalia polyphylla (DC.) Britton \& Rose & Tree & Damasceno-Jr,G.A. 1346 (COR 13708) & 1 & - \\
\hline $\begin{array}{l}\text { Senegalia } \text { cf. riparia (Kunth) Britton \& } \\
\text { Rose ex Britton \& Killip }\end{array}$ & Shrub & Damasceno-Jr,G.A. 1341 (COR 13711) & 1 & LC (IUCN) \\
\hline Senegalia tenuifolia (L.) Britton \& Rose & Shrub & $\begin{array}{l}\text { Damasceno-Jr,G.A. } 1351 \text { (COR 13706); } \\
\text { Takahasi, A, } 1112 \text { (COR 15200) }\end{array}$ & 1,2 & - \\
\hline $\begin{array}{l}\text { Stylosanthes acuminata M.B.Ferreira \& } \\
\text { Sousa Costa }\end{array}$ & Herb & $\begin{array}{c}\text { Takahasi, A. } 795 \text { (COR 15040); Takahasi, } \\
\text { A. } 844 \text { (15067); Takahasi, A. } 956 \text { (COR } \\
\text { 15124); Souza, N. } 10 \text { (COR 15808) }\end{array}$ & $3,4,6,9$ & - \\
\hline Stylosanthes capitata Vogel & Herb & Takahasi, A. 1108 (COR 15158) & 6 & - \\
\hline Stylosanthes guianensis (Aubl.) Sw. & Herb & Silva, R.R. 405 (UEC 141003) & 7 & - \\
\hline Stylosanthes montevidensis Vogel & Herb & Damasceno-Jr, G.A. 1319 (UEC 140180) & 1 & - \\
\hline Zornia cf. crinita (Mohlenbr.) Vanni & Herb & Damasceno-Jr, G.A. 4785 (CGMS 52405) & 1 & - \\
\hline Zornia latifolia Sm. & Herb & Silva, R.R 364 (COR 14650) & 7 & - \\
\hline Zornia reticulata $\mathrm{Sm}$. & Herb & $\begin{array}{l}\text { Culau, R. } 16 \text { (UEC 140174); Takahasi, A. } \\
802 \text { (COR 15042); Silva, R.R } 549 \text { (COR } \\
\text { 14651); Silva, R.H. } 908 \text { (CGMS 38782) }\end{array}$ & $1,3,7,8$ & - \\
\hline \multicolumn{5}{|l|}{ Iridaceae } \\
\hline Cipura formosa Ravenna & Herb & $\begin{array}{l}\text { Silva, R.H. } 949 \text { (CGMS 38805); Takahasi, } \\
\text { A. } 1181 \text { (COR 15233); Takahasi, A. } 1261\end{array}$ & $1,2,4$ & - \\
\hline \multicolumn{5}{|l|}{ Krameriaceae } \\
\hline Krameria grandiflora A. St. Hill & Herb & $\begin{array}{l}\text { Takahasi, A. 784; Takahasi, A. 808; Pott, } \\
\text { V.J. } 4780 \text { (CGMS 52708); Almeida, F.L.R. } \\
38 \text { (COR 15848) }\end{array}$ & $2,3,7,9$ & - \\
\hline
\end{tabular}


Flora in ironstone outcrops at the Urucum plateau

Continuation Table 2.

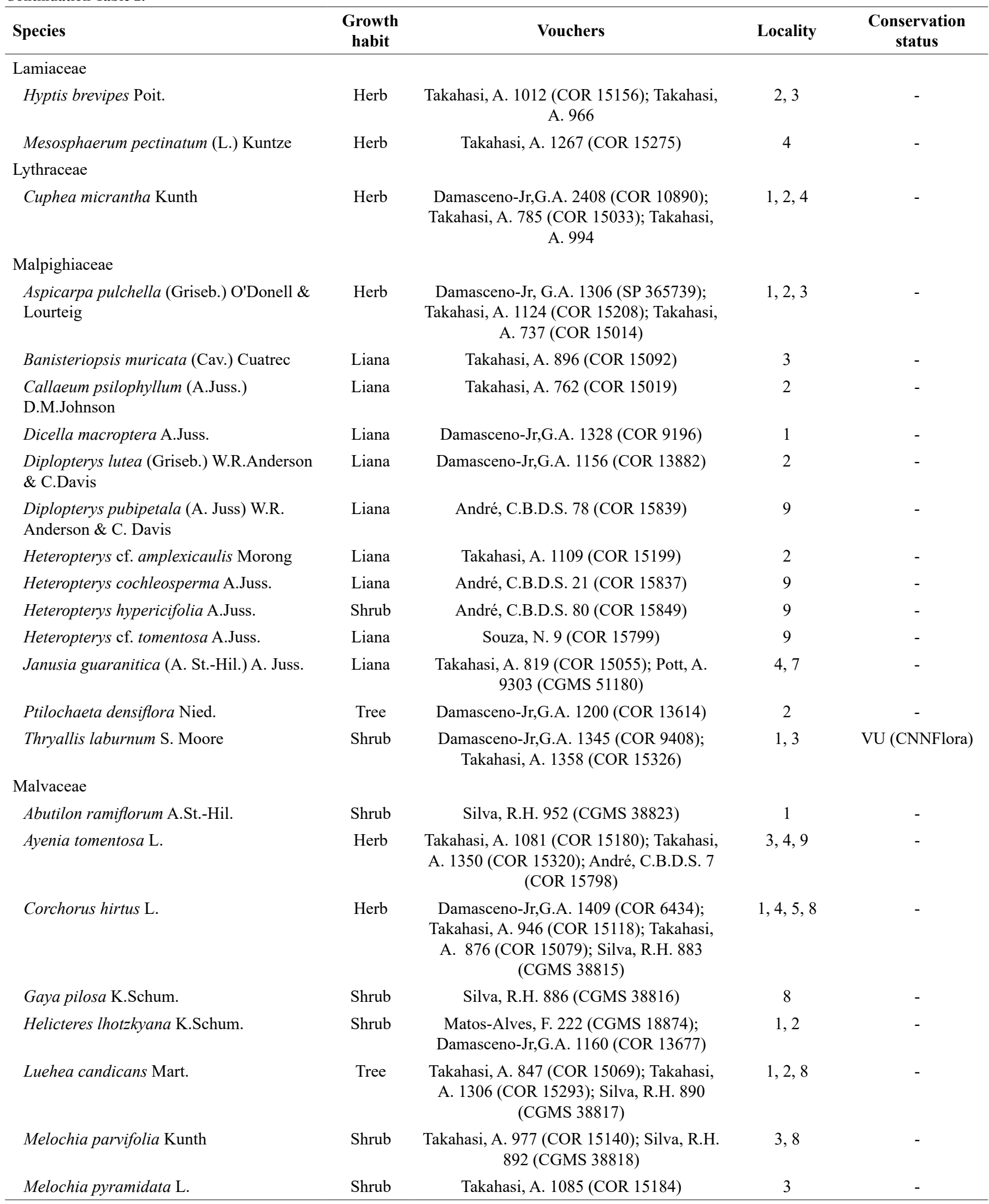


Lima, M.S. et al.

Continuation Table 2.

\begin{tabular}{|c|c|c|c|c|}
\hline Species & $\begin{array}{c}\text { Growth } \\
\text { habit }\end{array}$ & Vouchers & Locality & $\begin{array}{c}\text { Conservation } \\
\text { status }\end{array}$ \\
\hline Pavonia sidifolia Kunth & Herb & $\begin{array}{c}\text { Takahasi, A. } 919 \text { (COR 15102); Takahasi, } \\
\text { A. } 828 \text { (COR 15061); Silva, R.H. } 896 \\
\text { (CGMS 38819); Oliveira, P.P 329(COR } \\
\text { 15844) }\end{array}$ & $3,4,8,9$ & - \\
\hline $\begin{array}{l}\text { Pseudobombax marginatum (A.St.-Hil.) } \\
\text { A.Robyns }\end{array}$ & Tree & Takahasi, A. 1033 & 4 & - \\
\hline Sida cordifolia $\mathrm{L}$. & Herb & Takahasi, A. 1375 (COR 15341) & 4 & - \\
\hline Sida glomerata Cav. & Herb & Takahasi, A. 1281 (COR 15280) & 4 & - \\
\hline Sida linifolia Juss. ex Cav. & Herb & $\begin{array}{c}\text { Damasceno-Jr,G.A. } 1417 \text { (COR 2340); } \\
\text { Takahasi, A. } 1034 \text { (COR 15166); Takahasi, } \\
\text { A. } 1090 \text { (COR 15188); Oliveira, P.P } 205 \\
\text { (COR 15819); Silva, R.H. } 925 \text { (CGMS } \\
\text { 38826) }\end{array}$ & $1,3,4,9,10$ & - \\
\hline Waltheria indica $\mathrm{L}$. & Herb & Silva, R.H. 926 (CGMS 38827) & 10 & - \\
\hline Waltheria operculata Rose & Herb & $\begin{array}{c}\text { Takahasi, A. } 771 \text { (COR 15023); Takahasi, } \\
\text { A. } 711 \text { (COR 15002); Takahasi, A. } 944 \\
\text { (COR 15117) }\end{array}$ & $2,3,4$ & - \\
\hline Waltheria rotundifolia Schrank & Shrub & André, C.B.D.S. 6 (COR 15845) & 9 & - \\
\hline Wissadula macrantha R.E.Fr. & Shrub & $\begin{array}{l}\text { Damasceno-Jr, G.A. 1510; Takahasi, A. } \\
\text { 986 (COR 15143) }\end{array}$ & 1,4 & - \\
\hline \multicolumn{5}{|l|}{ Molluginaceae } \\
\hline Mollugo verticillata $\mathrm{L}$. & Herb & $\begin{array}{l}\text { Damasceno-Jr,G.A. } 1322 \text { (COR 13848); } \\
\text { Damasceno-Jr,G.A. } 1678 \text { (COR 13847); } \\
\text { Takahasi, A. } 788 \text { (COR 15036); Takahasi, } \\
\text { A. 839; Silva, R.H. } 895 \text { (CGMS 38833) }\end{array}$ & $1,2,3,4,8$ & - \\
\hline Eugenia punicifolia (Kunth) DC. & Shrub & Oliveira, P.P 204 (COR 15831) & 9 & - \\
\hline Eugenia pyriformis Cambess. & Shrub & $\begin{array}{c}\text { Takahasi, A. } 774 \text { (COR 5956); Takahasi, A. } \\
794 \text { (COR 15039) }\end{array}$ & 2,3 & - \\
\hline Myrcia laruotteana Cambess. & Shrub & Takahasi, A. 1264 (COR 15274) & 4 & - \\
\hline Myrcia pyrifolia (Desv.) Nied. & Shrub & Souza, N. 33 (COR 15287) & 9 & - \\
\hline Psidium guineense Sw. & Shrub & Silva, R.H. 922 (CGMS 38844) & 10 & - \\
\hline \multicolumn{5}{|l|}{ Nyctaginaceae } \\
\hline $\begin{array}{l}\text { Reichenbachia paraguayensis (D.Parodi) } \\
\text { Dugand \& Daniel }\end{array}$ & Tree & Damasceno-Jr,G.A. 1348 (COR 9200) & 1 & - \\
\hline \multicolumn{5}{|l|}{ Ochnaceae } \\
\hline Ouratea aff. castaneifolia (DC.) Engl. & Tree & $\begin{array}{c}\text { Takahasi, A. } 1301 \text { (COR 15288); Takahasi, } \\
\text { A. } 731\end{array}$ & 4,5 & - \\
\hline \multicolumn{5}{|l|}{ Onagraceae } \\
\hline Ludwigia lagunae (Morong) H. Hara & Shrub & Damasceno-Jr,G.A. 2414 (COR 10828) & 1 & - \\
\hline Ludwigia leptocarpa (Nutt.) H. Hara & Shrub & Silva, R.H. 936 (CGMS 38849) & 9 & - \\
\hline \multicolumn{5}{|l|}{ Orchidaceae } \\
\hline Cyrtopodium virescens Rchb. f. \& Warm. & Herb & $\begin{array}{c}\text { Takahasi, A. } 1114 \text { (COR 15370); } \\
\text { Damasceno-Jr, G.A. } 2783 \text { (CGMS 33893) }\end{array}$ & 3,6 & - \\
\hline
\end{tabular}


Continuation Table 2.

\begin{tabular}{|c|c|c|c|c|}
\hline Species & $\begin{array}{c}\text { Growth } \\
\text { habit }\end{array}$ & Vouchers & Locality & $\begin{array}{c}\text { Conservation } \\
\text { status }\end{array}$ \\
\hline \multicolumn{5}{|l|}{ Oxalidaceae } \\
\hline Oxalis frutescens $\mathrm{L}$. & Herb & $\begin{array}{c}\text { Takahasi, A. } 1126 \text { (COR 15210); Takahasi, } \\
\text { A. } 1106\end{array}$ & 2,6 & - \\
\hline Passiflora foetida $\mathrm{L}$. & Liana & Takahasi, A. 1402 & 6 & - \\
\hline Piriqueta corumbensis Moura & Herb & Takahasi, A. 1052 (COR 15176) & 3 & - \\
\hline Piriqueta morongii Rolfe & Herb & Culau, R. 18 (COR 13702) & 1 & - \\
\hline Turnera $\mathrm{cf}$. blanchetiana Urb. & Herb & Damasceno-Jr,G.A. 3299 (COR 13150) & 1 & - \\
\hline Turnera grandiflora (Urb.) Arbo & Herb & $\begin{array}{l}\text { Takahasi, A. 663; Takahasi, A. } 897 \text { (COR } \\
\text { 15093); Takahasi, A. } 1260 \text { (COR 15272); } \\
\text { Damasceno-Jr,G.A. } 2791 \text { (COR 10914); } \\
\text { André, C.B.D.S. } 13 \text { (COR 15809) }\end{array}$ & $1,3,4,6,9$ & - \\
\hline $\begin{array}{l}\text { Turnera melochioides A. St.-Hil. \& } \\
\text { Cambess. }\end{array}$ & Herb & Oliveira, P.P 206 (COR 15841) & 9 & - \\
\hline Turnera pumilea $\mathrm{L}$. & Herb & $\begin{array}{c}\text { Takahasi, A. } 820 \text { (COR 15056); Takahasi, } \\
\text { A. } 1381 \text { (COR 15343) }\end{array}$ & 4,6 & - \\
\hline Turnera weddelliana Urb. \& Rolfe & Herb & $\begin{array}{c}\text { Damasceno } 1419 \text { (COR 13630); } \\
\text { Damasceno-Jr,G.A. } 1687 \text { (COR 13698) }\end{array}$ & 1,2 & - \\
\hline \multicolumn{5}{|l|}{ Phyllanthaceae } \\
\hline Phyllanthus orbiculatus Rich. & Herb & $\begin{array}{c}\text { Takahasi, A. } 1253 \text { (COR 12394); Silva, } \\
\text { R.H. } 897 \text { (CGMS } 38852 \text { ); Almeida, L.W. } \\
45 \text { (COR 15815) }\end{array}$ & $4,8,9$ & - \\
\hline Axonopus pressus (Steud.) Parodi & Herb & Oliveira, P.P 324 (COR 15821) & 9 & - \\
\hline $\begin{array}{l}\text { Axonopus suffultus (J.C.Mikan ex Trin.) } \\
\text { Parodi }\end{array}$ & Herb & Takahasi, A. 952 (COR 11752) & 6 & - \\
\hline Chloris elata Desv. & Herb & Takahasi, A. 796 (COR 11716) & 3 & - \\
\hline Dactyloctenium aegyptium (L.) Willd. & Herb & Takahasi, A. 1207 (COR 11715) & 2 & - \\
\hline Digitaria bicornis (Lam.) Roem. \& Schult. & Herb & Takahasi, A. 1293 (COR 11717) & 3 & - \\
\hline Digitaria insularis (L.) Mez ex Ekman & Herb & $\begin{array}{l}\text { Damasceno-Jr, G.A. 1412; Oliveira, P.P } \\
325 \text { (COR 15796) }\end{array}$ & 1,9 & - \\
\hline Digitaria sanguinalis (L.) Scop. & Herb & Silva, R.H. 884 (CGMS 38855) & 8 & - \\
\hline Eragrostis articulata (Schrank) Nees & Herb & Silva, R.H. 885 (CGMS 38856) & 8 & - \\
\hline Eragrostis orthoclada Hack. & Herb & Takahasi, A. 1290 (CGMS 20374) & 3 & LC (IUCN) \\
\hline Eragrostis pilosa (L.) P.Beauv. & Herb & Silva, R.H. 933 (CGMS 38875) & 9 & - \\
\hline Eustachys distichophylla (Lag.) Nees & Herb & Takahasi, A. 1272 (COR 11721) & 4 & - \\
\hline Gouinia latifolia (Griseb.) Vasey & Herb & $\begin{array}{l}\text { Damasceno-Jr, G.A. 1416; Takahasi, A. } \\
1021 \text { (COR 11739) }\end{array}$ & 1,3 & - \\
\hline Leptochloa virgata (L.) P.Beauv. & Herb & Silva, R.H. 889 (CGMS 38867) & 8 & - \\
\hline
\end{tabular}


Lima, M.S. et al.

Continuation Table 2.

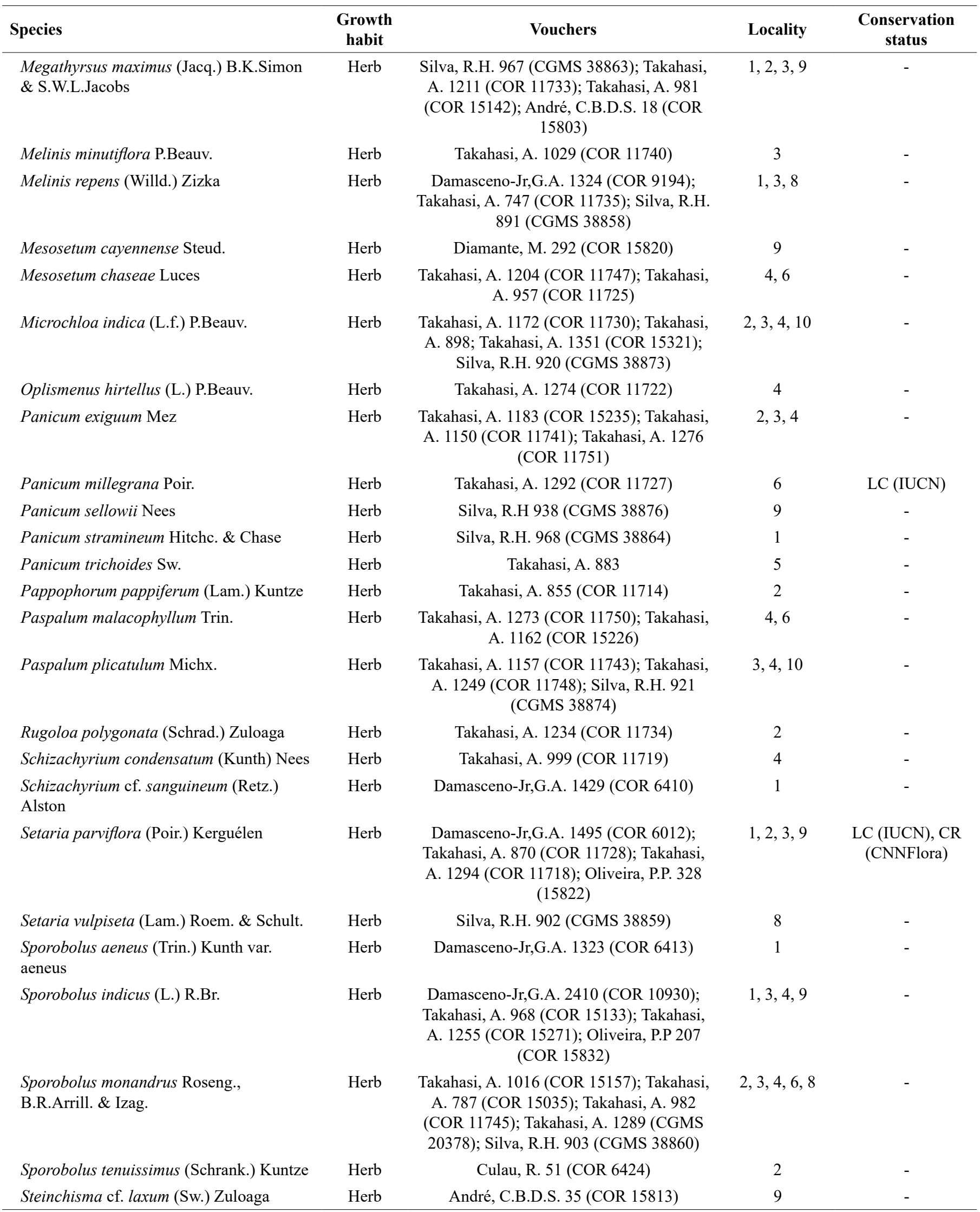


Continuation Table 2.

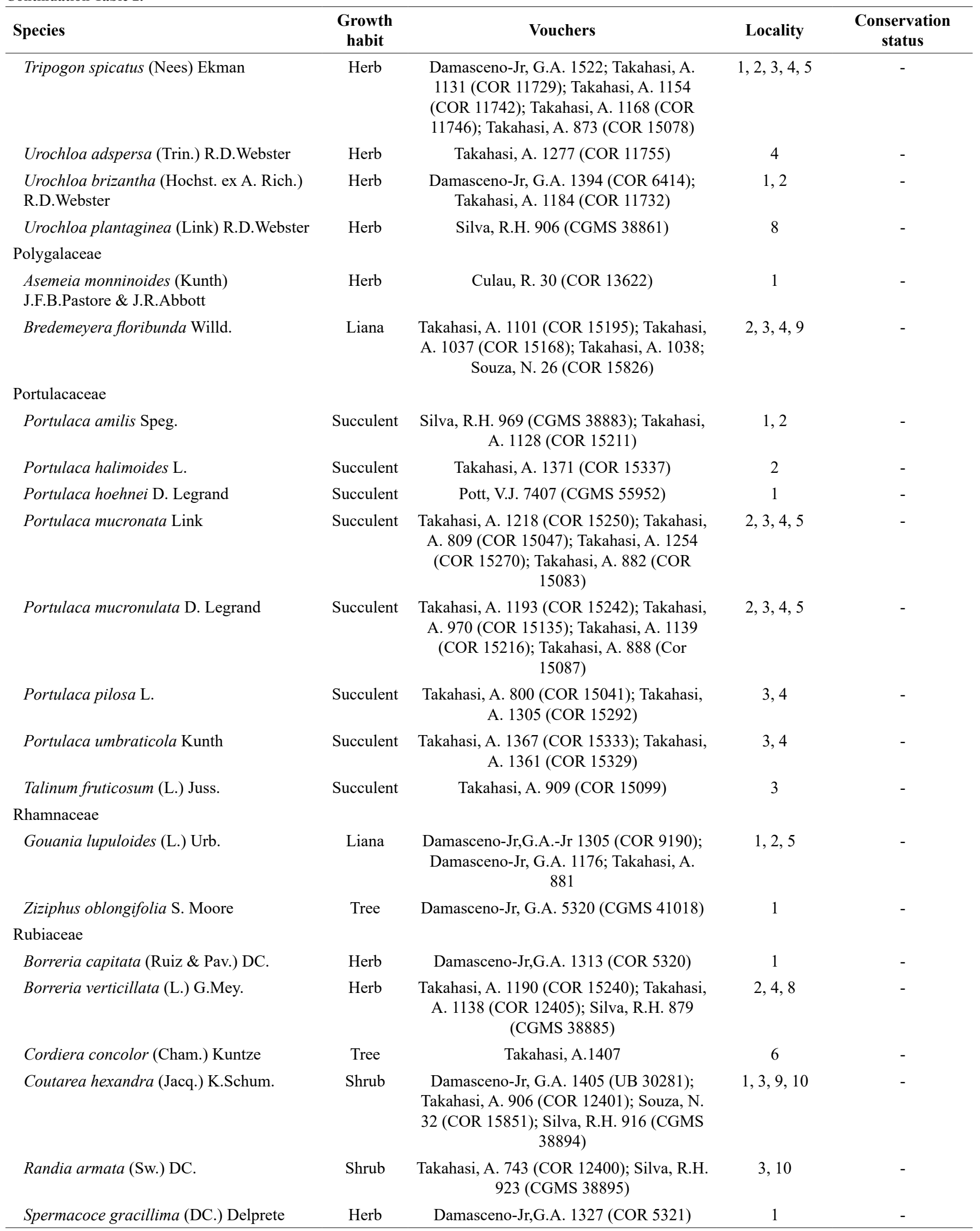


Lima, M.S. et al.

Continuation Table 2.

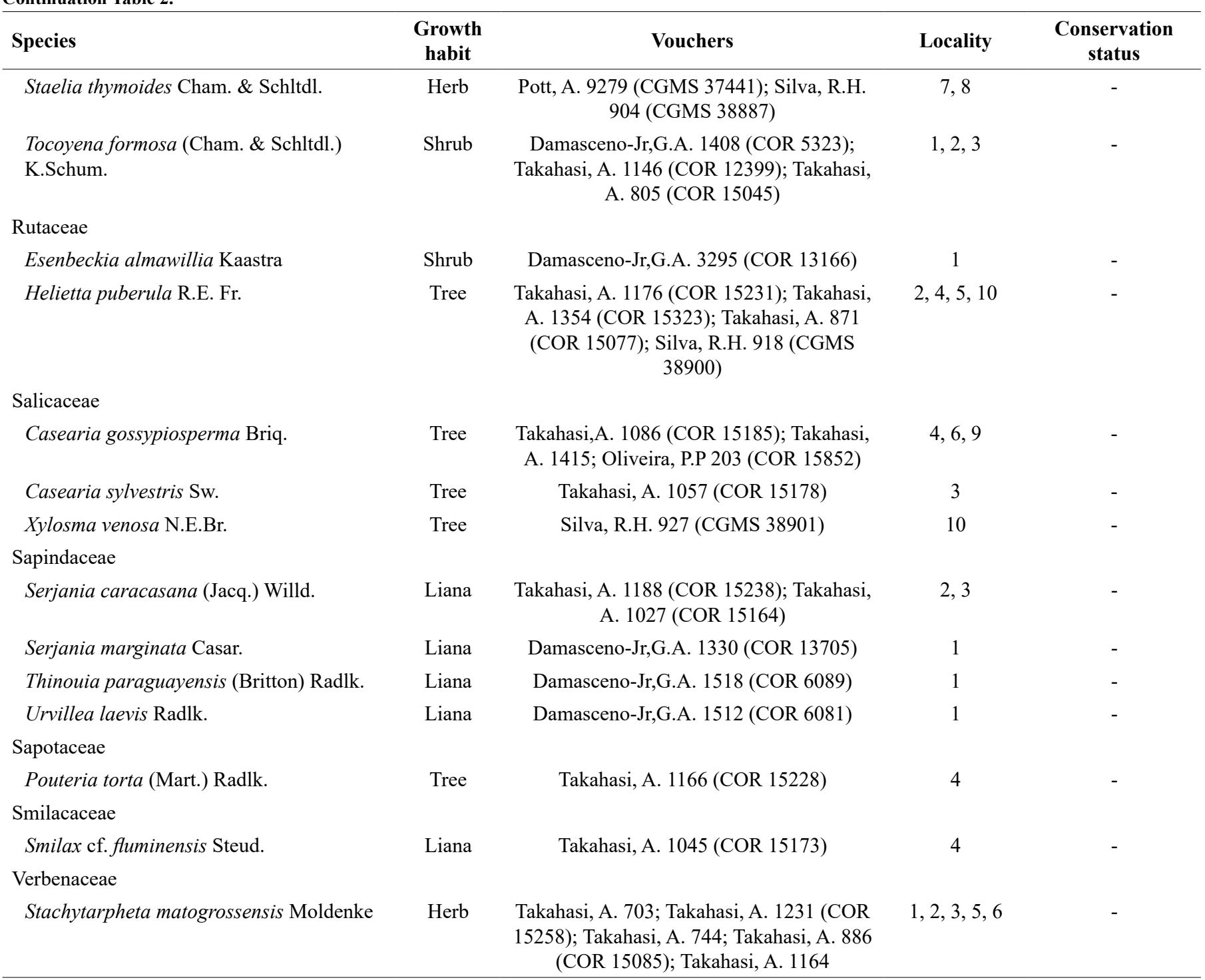

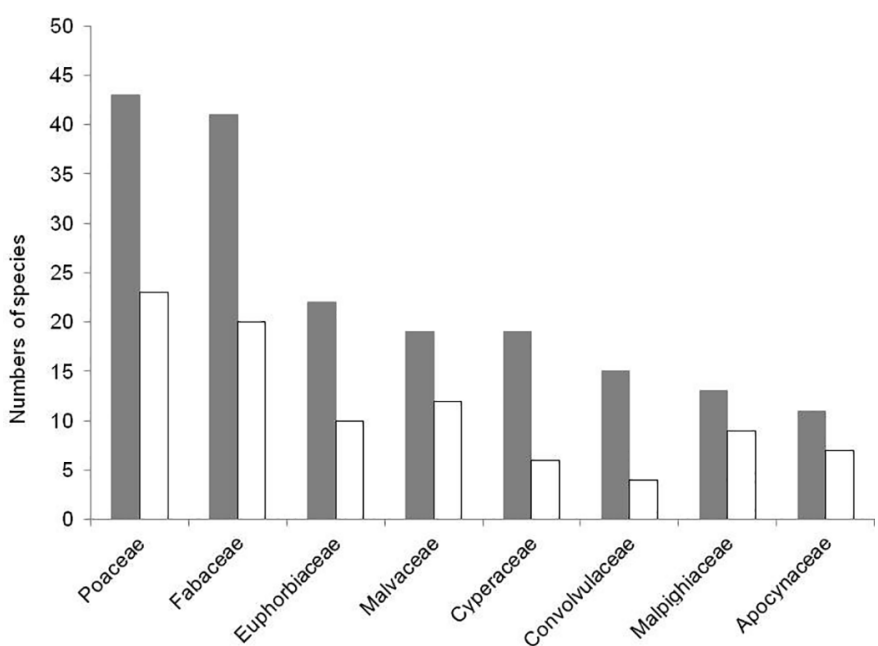

Figure 2. Number of species (gray bar) and genera (white bar) of the richest families found on the cangas at the Urucum Plateau, Western Border of Pantanal, MS.
E. Holzh. and Borreria verticillata (L.) G.Mey. In the seasonally wet areas we can find species such as Ludwigia lagunae (Morong) H. Hara, Crotalaria pallida Aiton, Sporobolus indicus (L.) R.Br. and Eleocharis contracta Maury ex Micheli.

Based on the species list of Flora do Brasil 2020, among the total number of recorded species, 177 (58.61\%) are of wide distribution, occurring in almost all regions in Brazil, such as Acrocomia aculeata (Jacq.) Lodd. ex Mart., Anadenanthera colubrina (Vell.) Brenan, Bromelia balansae Mez, Conyza bonariensis (L.) Cronquist, Handroanthus impetiginosus (Mart. ex DC.) Mattos, Myracrodruon urundeuva Allemão, Ludwigia leptocarpa (Nutt.) H. Hara and Urochloa brizantha (Hochst. ex A. Rich.) R.D.Webster. Other species are recorded as common in other biomes, such as Myrcia pyrifolia (Desv.) Nied. (Amazon Rainforest), Sida coradinii Krapov. and Zephyranthes cearensis (Herb.) Baker (Caatinga), Aspidosperma quirandy Hassl. (Atlantic Forest), Frailea cataphracta (Dams) Britton $\&$ Rose, Digitaria sanguinalis (L.) Scop. and Staelia thymoides Cham. \& Schltdl. (Pampas). 
Twenty species are listed in the International Union Conservation of Nature and Natural Resources (IUCN 2018) as endangered species, and nine species in the Red Book of Brazilian flora (Martinelli \& Moraes 2013), (Table 2). The species Muellera variabilis (RR.Silva \& AMG.Azevedo) MJ.Silva \& AMG.Azevedo, Gomphrena centrota E. Holzh., Bonamia balansae Hallier f. and Bauhinia leptantha Malme are considered as rare species because of their restricted distribution or the scarcity of information on the size of their populations (Giulietti et al. 2009). Two species are considered endemic to the ironstone outcrops at the Urucum Plateau: G. centrota (Tomás et al. 2010) and Discocactus ferricola Buining \& Brederoo, being the latter also present in Morro do Mutum, Bolivia (Tomás et al. 2010, Takahasi \& Meirelles 2014).

The herbaceous habit was the predominant growth form on ironstone outcrops in the Urucum Plateau (47.02\%), probably due to the high species richness in the families Poaceae and Cyperaceae, and the high frequency of herbaceous species in Malvaceae, Fabaceae and Passifloraceae (Figure 3). Shrub (21.85\%) and arboreal species (10.26\%) were also common in these areas, with great contribution of Fabaceae, Euphorbiaceae, Malvaceae, Myrtaceae and Apocynaceae.

Lianas $(13,91 \%)$ were represented by the families Convolvulaceae, Malpighiaceae and Apocynaceae mainly (Figure 3). Although succulent species represent only $6.29 \%$ of the richness of the local flora, they are quite conspicuous in the landscape, forming dense mats of vegetation composed of Bromelia balansae Mez, Deuterochnonia meziana Kuntze ex Mez, Opuntia retrorsa Speg. and rupicolous vegetation of Portulaca sp.

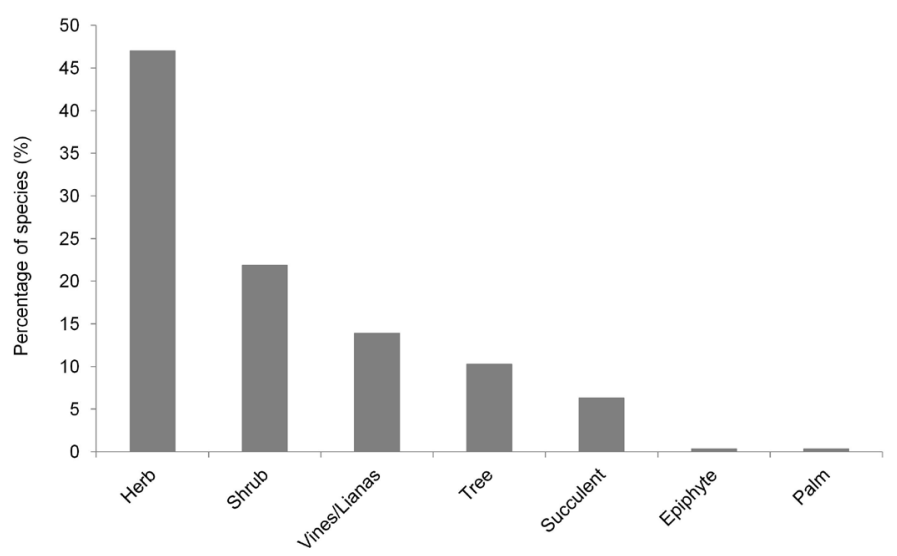

Figure 3. Growth habit of the species found on canga areas at the Urucum Plateau, Western Border of Pantanal, MS.

Considering the floristic associations present on the rocky outcrops at the Urucum plateau, we can distinguish ecological relationships between vegetation and edaphic variables, such as Gomphrena centrota E. Holzh., Discocactus ferricola Buining \& Brederoo and Polycarpaea corymbosa (L.) Lam., that lodge directly on the hardened substrate, on slopes or in small cavities and fissures, covering rocky substrates; and soil islands, generally formed by mats of Bromelia balansae Mez and Deuterocohnia meziana Kuntze ex Mez, containing in their interior trees or shrubs of Acrocomia aculeata (Jacq.) Lodd. ex Mart., Bauhinia pentandra (Bong.) Vogel ex Steud., Commiphora leptophloeos (Mart.) J.B. Gillett, Myracrodruon urundeuva Allemão and Tocoyena formosa (Cham. \& Schltdl.) K.Schum., among others (Takahasi 2010, Takahasi
\& Meirelles 2014, Takahasi 2015; Figure 4 F). At the edges of soil islands, there are also many herbaceous and annual species, such as Borreria capitata (Ruiz \& Pav.) DC., Calea rupicola Chodat, Mimosa bimucronata (DC.) Kuntze, M. xantocentra Mart. and Waltheria operculata Rose, which desiccate completely during the dry season. Other species such as Portulaca mucronata Link, Cipura formosa Ravenna and Zephyranthes cearensis (Herb.) Baker occur in seasonally flooded sites at topographically lower areas (Takahasi \& Meirelles 2014, Takahasi 2015, Figure 4 C).

About $58.28 \%$ of the species were found in only one of the ironstone outcrops at the Urucum Plateau, and none of the species was recorded in all 10 analyzed areas. The similarity índex (Table 3 ) in the composition of the angiosperm flora between the ironstone outcrops was low and ranged from 0 (Rabicho farm, P8 and the Municipal Natural Park Piraputangas, P10) to $41 \%$ (Pantanal Park Road, P1 and Band'alta farm, P2).

By crossing data contained in SpeciesLink (CRIA 2018), Flora do Brasil 2020 (2018), Jabot (2018) and checklists of Flora from Mato Grosso do Sul state (Pott \& Pott 1996, Schutz 2014, Profice et al. 2015, Machate et al. 2016, Araújo \& Trevisan 2018, Barbosa 2018, Bortolotto et al. 2018, Damasceno-Junior et al. 2018, Farinaccio \& Simões 2018, Fiaschi 2018, Francener et al. 2018, Groppo et al. 2018, Guglieri-Caporal et al. 2018, Lobão et al. 2018, Loiola \& Cordeiro 2018, Panfiglio et al. 2018, Proença et al. 2018, Roque et al. 2018, Sartori et al. 2018, Secco et al. 2018, Versieux et al. 2018, Zappi et al. 2018), it was verified that from the species presented here, 27 were not previously recorded for Mato Grosso do Sul and three species have no information of occurrence in Brazil (Table 4).

\section{Discussion}

Previous works on floristics, phytosociology and/or phenology indicated the occurrence of 243 species distributed in 66 botanical families, including pteridophytes (Takahasi 2010, Takahasi \& Meirelles 2014, Takahasi 2015, Oliveira 2016), not included in this study. Thus, this study added 59 new occurrences of species of angiosperms to the ironstone outcrops flora in the Urucum Plateau. The high richness of species found, similar to other ferruginous outcrops areas (Jacobi et al. 2007, Mourão \& Stehmann 2007, Viana \& Lombardi 2007, Pifano et al. 2010, Jacobi \& Carmo 2008b, Jacobi \& Carmo 2011, Carmo \& Jacobi 2013, Skirycz et al. 2014), may be related to the proximity of seasonal forest patches bordering these formations, and related to the existence of cavities that allow the accumulation of soil and water pools, resulting in microhabitats that enable a variety of plant associations (Jacobi \& Carmo 2008a, Takahasi \& Meirelles 2014).

The high turnover of species between the areas of ironstone outcrops sampled, together with the seasonality, where some species are only perceived during the rainy season, help to explain the high richness found in these environments. Another factor that contributes to species richness is the presence of subspontaneous and ruderal species from adjacent pastures, such as Megathyrsus maximus (Jacq.) B.K.Simon \& S.W.L.Jacobs and Melinis minutiflora P.Beauv.

Overall, species are established according to the topographic characteristics in the environment and the substrate granulometry, so that the structure and spatial-temporal distribution of the species are not homogeneous on ironstone outcrops (Takahasi \& Meirelles 2014). 

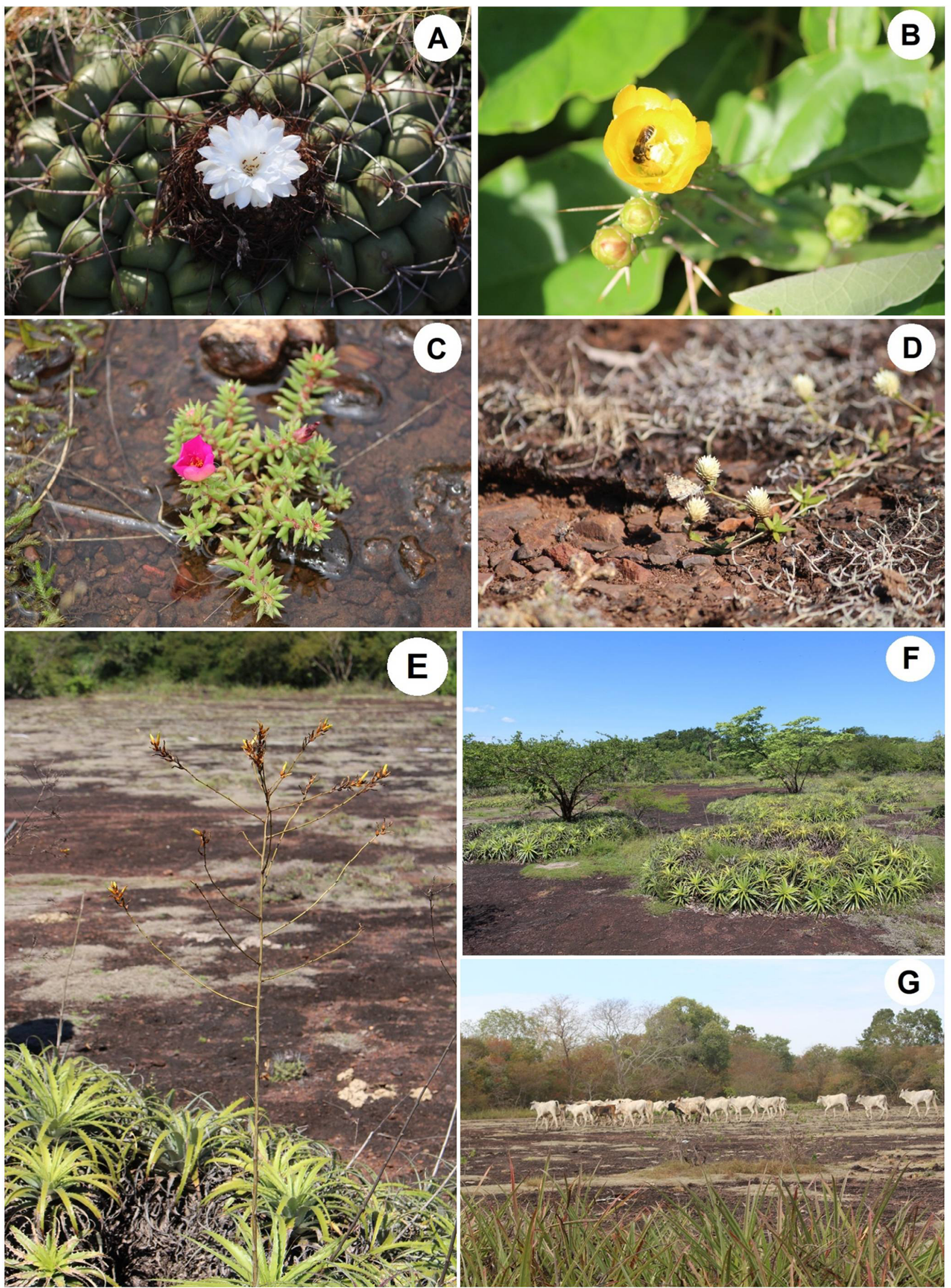

Figure 4. Species from the cangas plant community at the Urucum Plateau. A) Discocactus ferricola, B) Opuntia retrorsa, C) Portulaca mucronata in a flooded rocky substrate área, canga in the Band'alta farm, D) Gomphrena centrota, endemic species of the region, E) Deuterocohnia meziana. Cangas environments (Band'alta farm): F) Soil islands with densification of Deuterocohnia meziana and arboreal-shrub species in the center. In the background, seasonal forests bordering the rocky substrates, G) the vulnerability of the cangas of the region against anthropic pressure, e.g. by grazing livestock. 
Flora in ironstone outcrops at the Urucum plateau

Table 3. Sorensen index among the cangas (P1-P10) at the Urucum Plateau, Western Border of Pantanal, MS. The numbers of localities are shown in Table 1.

\begin{tabular}{|c|c|c|c|c|c|c|c|c|c|c|}
\hline & P1 & P2 & P3 & P4 & P5 & P6 & P7 & P8 & P9 & P10 \\
\hline $\mathrm{P} 1$ & 1 & 0.418 & 0.333 & 0.236 & 0.176 & 0.095 & 0.118 & 0.143 & 0.127 & 0.063 \\
\hline $\mathrm{P} 2$ & & 1 & 0.384 & 0.317 & 0.200 & 0.155 & 0.108 & 0.134 & 0.093 & 0.066 \\
\hline $\mathrm{P} 4$ & & & & 1 & 0.210 & 0.205 & 0.133 & 0.165 & 0.175 & 0.124 \\
\hline P5 & & & & & 1 & 0.069 & 0.043 & 0.120 & 0.030 & 0.053 \\
\hline P8 & & & & & & & & 1 & 0.085 & 0 \\
\hline P9 & & & & & & & & & 1 & 0.068 \\
\hline P10 & & & & & & & & & & 1 \\
\hline
\end{tabular}

Table 4. List of species first mentioned for the state of Mato Grosso do Sul and for Brazil.

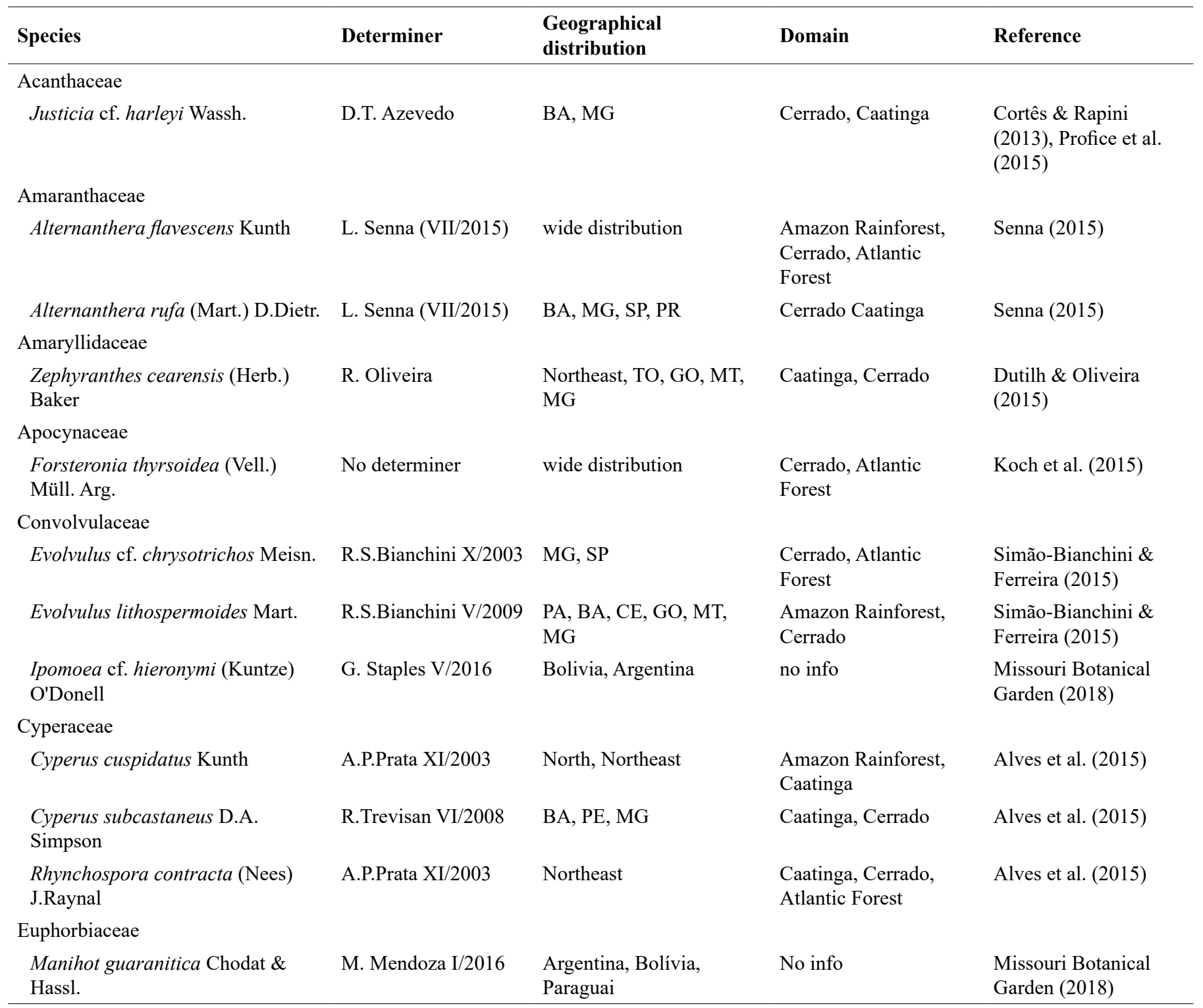


Lima, M.S. et al.

Continuation Table 4.

\begin{tabular}{|c|c|c|c|c|}
\hline Species & Determiner & $\begin{array}{l}\text { Geographical } \\
\text { distribution }\end{array}$ & Domain & Reference \\
\hline \multicolumn{5}{|l|}{ Fabaceae } \\
\hline \multicolumn{5}{|l|}{ Iridaceae } \\
\hline \multicolumn{5}{|l|}{ Lamiaceae } \\
\hline $\begin{array}{l}\text { Mesosphaerum cf. pectinatum (L.) } \\
\text { Kuntze }\end{array}$ & No determiner & $\begin{array}{l}\text { Northeast, Sudeste, PA, } \\
\text { PR, SC }\end{array}$ & $\begin{array}{l}\text { Amazon Rainforest, } \\
\text { Caatinga, Cerrado, } \\
\text { Atlantic Forest }\end{array}$ & Harley et al. (2015) \\
\hline \multicolumn{5}{|l|}{ Malvaceae } \\
\hline Waltheria operculata Rose & G.L.Esteves X/2009 & $\begin{array}{l}\text { MT, GO, BA, PB, PE, } \\
\text { RN,MG, RJ }\end{array}$ & $\begin{array}{l}\text { Caatinga, Cerrado, } \\
\text { Atlantic Forest, } \\
\text { Pantanal }\end{array}$ & Esteves (2015) \\
\hline Waltheria rotundifolia Schrank & P.P.Oliveira & BA, PE, PI, RN & Caatinga, Cerrado & Esteves (2015) \\
\hline \multicolumn{5}{|l|}{ Myrtaceae } \\
\hline Myrcia pyrifolia (Desv.) Nied. & No determiner & $\mathrm{PA}, \mathrm{AC}, \mathrm{AM}$ & Amazon Rainforest & Sobral et al. (2015) \\
\hline \multicolumn{5}{|l|}{ Poaceae } \\
\hline Digitaria sanguinalis (L.) Scop. & R.H.Silva III/2013 & $\mathrm{RS}$ & Pampa & Canto-Dorow (2015) \\
\hline $\begin{array}{l}\text { Sporobolus tenuissimus (Schrank.) } \\
\text { Kuntze }\end{array}$ & T.S.Filgueiras X/2001 & wide distribution & $\begin{array}{l}\text { Amazon Rainforest, } \\
\text { Caatinga, Cerrado, } \\
\text { Atlantic Forest }\end{array}$ & Longhi-Wagner (2015) \\
\hline Portulaca halimoides L. & $\begin{array}{l}\text { A.O.P.Coelho } \\
\text { VI/2010 }\end{array}$ & $\begin{array}{l}\text { Northeast, AP, PA, RR, } \\
\text { TO, DF, GO, ES, MG, RJ }\end{array}$ & $\begin{array}{l}\text { Amazon Rainforest, } \\
\text { Caatinga, Cerrado, } \\
\text { Atlantic Forest }\end{array}$ & $\begin{array}{l}\text { Coelho \& Zappi } \\
(2015)\end{array}$ \\
\hline Portulaca hoehnei D. Legrand & A.O.P.Coelho X/2004 & MS & No info & $\begin{array}{l}\text { Missouri Botanical } \\
\text { Garden (2018) }\end{array}$ \\
\hline Portulaca mucronulata D. Legrand & $\begin{array}{l}\text { A.O.P.Coelho } \\
\text { VI/2010 }\end{array}$ & Argentina & No info & $\begin{array}{l}\text { Missouri Botanical } \\
\text { Garden (2018) }\end{array}$ \\
\hline \multicolumn{5}{|l|}{ Rubiaceae } \\
\hline $\begin{array}{l}\text { Spermacoce gracillima (DC.) } \\
\text { Delprete }\end{array}$ & P.Delpetre 1998 & $\mathrm{GO}, \mathrm{TO}$ & Cerrado & Cabral e Salas (2015) \\
\hline
\end{tabular}


The topographic development of these formations resulted in unique habitats such as fissures, cavities, small temporary ponds, depressions and exposed rocks, which differ from adjacent landscapes, constituting refuges for species adapted to xeric and mesic conditions (Jacobi et al. 2007, Jacobi \& Carmo 2008b).

During the driest season of the year, most of the ironstone outcrops species present intense deciduousity, assuming a similar xeric aspect to that found in other ferruginous geosystems in Brazil (Mota et al. 2015). The difficulty for soil drainage, associated with climatic conditions in the region, gave rise to a prickly vegetation, physiognomically similar to the Caatinga, adapted to extreme conditions of evapotranspiration, thermal amplitude, underdeveloped soil, presence of heavy metals and drought periods in the region (Loureiro et al. 1982, Jacobi \& Carmo 2008a, Jacobi et al. 2015). These adaptations may involve partial or total loss of aerial structures in the dry season, succulence, pseudo-bulbs in orchids, clonal reproduction, imbricate leaves, slow growth and CAM photosynthesis in order to resist desiccation cycles and subsequent rehydration (Jacobi et al. 2007, Jacobi \& Carmo 2008b, Skirycz et al. 2014, Jacobi et al. 2015).

The predominance of species of wide distribution differs from that expected for ironstone outcrops in other regions of Brazil, where there are occurrences of exclusive species and different species from neighboring ecosystems (Skirycz et al. 2014). The deciduous forests in the Urucum Plateau that border the ironstone outcrops are characterized by the confluence of species from different biomes that surround it, keeping floristic relationships with the Bosque Seco Chiquitano in Bolivia (Jardim et al. 2003), and representatives from Chaco, Cerrado, Amazon Rainforest, Atlantic Forest and Southern Forests in the south and east (Rizzini 1997, Pott \& Pott 2003, Tomás et al. 2010). In addition, it presents common species to the Caatinga, a vestige of a vegetative distribution pattern of the Pleistocene period. The period was characterized by a dry climate which resulted in the expansion of the seasonal forests and the retreat of the humid forests, allowing the connection of the previously disjunct vegetation of Northeast and Midwest regions of Brazil and Argentina (Prado \& Gibbs 1993, Linares-Palomino et al. 2003).

The high proportion of herbaceous and arboreal-shrub species is explained by the representativeness of the families Fabaceae and Poaceae, similar to the results found for other plant communities in ferruginous outcrops in Brazil (Mourão \& Stehmann 2007, Viana \& Lombardi 2007, Pifano et al. 2010, Jacobi \& Carmo 2011, Carmo \& Jacobi 2013, Skirycz et al. 2014). On the other hand, the family Asteraceae, that is well represented in ironstone outcrops of other regions of the country (Mourão \& Stehmann 2007, Viana \& Lombardi 2007, Pifano et al. 2010, Ataíde et al. 2011), presented low species richness in the studied region. According to Rizzini (1997), the cangas are hardened ferrous formations and therefore have discontinuous vegetation and are typically covered by very specialized herbaceous vegetation, while on its edge woody species remain. The families Poaceae and Fabaceae are not abundant only on ironstone outcrops of the Urucum Plateau, but also among the herbaceous and woody groups, and in general between Angiosperms in Brazil (Forzza et al. 2010, BFG 2015).
Sørensen's similarity estimates found between the areas were low, with values below 0.5 , which corroborates other studies indicating high beta diversity for these phytophysiognomies (Mourão \& Stehmann 2007, Jacobi \& Carmo 2008b, Pifano et al. 2010, Messias et al. 2012). The areas of Pantanal Park Road and Band'alta farm are the closest to each other, which justifies the highest similarity found. However, it should be noted that due to the species surveying method used in this study, sampling effort among the ironstone outcrops was not uniform. Thus, some common and abundant species may not have been listed for some of the ironstone outcrops because they represent species already cataloged for the environment, this way reducing similarity between some areas. In rupestrian grassland on ferruginous outcrops in Iron Quadrangle (Jacobi \& Carmo 2008b) low proportion (5\%) of common species was found among the analyzed areas, suggesting high beta diversity, probably resulting from the topographic and microclimatic variations in the habitat between areas.

Recent studies have updated the occurrence of some of the species recorded here for Mato Grosso do Sul such as Tradescantia boliviana (Hassk.) J.R.Grant (Pellegrini et al. 2017), Deuterohconia meziana Kuntze ex Mez (Schutz 2014), Dyckia excelsa Leme (Paggi et al. 2015) and Tarenaya eosina (J.F.Macbr.) Soares Neto \& Roalson, a species considered endemic to Paraguay (Costa-e-Silva 2000, Soares-Neto et al. 2018). Portulaca hoehnei D. Legrand is not a species of occurrence for Brazil, although its holotype is from the region of Corumbá (Hoehne $R$ 3565). New occurrences of species in the ironstone outcrops of the Urucum Plateau demonstrate the need for continuous research to update the knowledge about the size and distribution of the species that occur in these formations, and the level of conservation in which they are found.

Although the ironstone outcrops in the Urucum Plateau do not directly undergo mineral extractive activity, due to their location at the foothill and relative distance of the mines of iron and manganese extraction, these environments are under intense anthropic action. Extraction of ornamental species such as orchids, bromeliads and cacti for sale at local fairs, grazing by cattle, fire action and removal of local vegetation by owners are pressures undergone by the flora from these areas (Takahasi \& Meirelles 2014, Figure 4 G).

Even in a situation of maximum vulnerability, less than $1 \%$ of canga areas in Brazil are included in Full Protected Conservation Units, such as National and State Parks (Carmo et al. 2012). The high diversity and presence of endemic, rare, endangered and/or not yet cataloged species for the Urucum Plateau region, reinforces the need for local conservation units that can guarantee the preservation of these species, since the environmental protection areas in the region are insufficient to guarantee the maintenance of typical populations from this habitat (Tomás et al. 2010). In addition, our data can significantly contribute for changing the conservation status of some species, since only $13 \%$ of the rare species present in ferruginous geosystems from Brazil are cited in the List of Endangered Species, in large part due to insufficient data for this type of environment (Giulietti et al. 2009, Jacobi et al. 2015). 


\section{Acknowledgments}

We thank CAPES for the financial assistance and the $\mathrm{PhD}$ scholarship granted to the first author, to CNPq for the grants to Geraldo Alves Damasceno Junior and to Andréa C. Araujo (310999/2018-9), the Instituto Federal de Mato Grosso do Sul for supporting this research, the Missão Salesiana for permitting the access to Band'alta ranch, and the owners of the other farms for allowing our access to the study sites, to the herbaria where the collectings are deposited.

\section{Author Contributions}

Michele Soares de Lima: Substantial contribution in the concept and design of the study, contribution to data collection, contribution to data analysis and interpretation, contribution to manuscript preparation, contribution to critical rrevision, adding intelectual content.

Adriana Takahasi: Substantial contribution in the concept and design of the study, contribution to data collection, contribution to data analysis and interpretation, contribution to manuscript preparation, contribution to critical rrevision, adding intelectual content.

Geraldo Alves Damasceno-Junior: Substantial contribution in the concept and design of the study, contribution to data collection, contribution to data analysis and interpretation, contribution to manuscript preparation, contribution to critical rrevision, adding intelectual content.

Andréa Cardoso Araujo: Substantial contribution in the concept and design of the study, contribution to data analysis and interpretation, contribution to manuscript preparation, contribution to critical rrevision, adding intelectual content.

\section{Conflicts of interest}

The authors declare that they have no conflict of interest related to the publication of this manuscript.

\section{References}

ALVES, M., HEFLER, S.M., TREVISAN, R., SILVA-FILHO, P.J.S. \& RIBEIRO, A.R.O. 2015. Cyperaceae. In Lista de Espécies da Flora do Brasil. Jardim Botânico do Rio de Janeiro. Available in: $<$ http://floradobrasil.jbrj.gov.br/jabot/floradobrasil/FB17141>. Access in: Jul 26th, 2018.

APG. 2016. An update of the Angiosperm Phylogeny Group classification for the orders and families of flowering plants: APG IV. Botanical Journal of the Linnean Society 181:1-20.

ARAÚJO, A.C. \& TREVISAN, R. 2018. Cyperaceae da flora Sulmatogrossense: composição florística. Iheringia, Sér. Bot., 73(supl.):190-200.

ATAÍDE, E.S., CASTRO, P.T.A. \& FERNANDES, G.W. 2011. Florística e caracterização de uma área de campo ferruginoso no Complexo Minerário Alegria, Serra de Antônio Pereira, Ouro Preto, Minas Gerais, Brasil. Rev. Árvore, 35(6):1265-1275.

BARBOSA, M.R.V. 2018. Check-list das Rubiaceae do Estado de Mato Grosso do Sul, Brasil. Iheringia, Sér. Bot., 73(supl.):335-341.

BORGES, C.A., WERLE, H.J.S., ROSA, D.B., PAIVA, D.J., MORAES, E.P. \& SILVA, L.B.S.M. 1997. Geomorfologia. In BRASIL. Ministério do Meio Ambiente, dos Recursos Hidrícos e da Amazônia Legal. Plano de Conservação da Bacia do Alto Paraguai (Pantanal) - PCBAP. Diagnóstico dos meios físico e biótico.MMA/SEMAM/ PNMA, Brasília, v.2, t.3, p.73-119.
BORTOLOTTO, I.M., DAMASCENO-JUNIOR, G.A. \& POTT, A. 2018. Lista preliminar das plantas alimentícias nativas de Mato Grosso do Sul, Brasil. Iheringia, Sér. Bot., 73(supl.):101-116.

BOVINI, M.G. 2015. Sida. In Lista de Espécies da Flora do Brasil. Jardim Botânico do Rio de Janeiro. Available in: <http://floradobrasil.jbrj. gov.br/jabot/floradobrasil/FB105575>. Access in: Jul 26th, 2018.

BRAZIL FLORA GROUP BFG. 2015. Growing knowledge: an overview of Seed Plant diversity in Brazil. Rodriguésia 66(4): 1085-1113.

CABRAL, E. \& SALAS, R. 2015. Spermacoce. In Lista de Espécies da Flora do Brasil. Jardim Botânico do Rio de Janeiro. Available in: <http://floradobrasil.jbrj.gov.br/jabot/floradobrasil/FB39374>. Access in: Jul 26th, 2018.

CANTO-DOROW, T.S. 2015. Digitaria. In Lista de Espécies da Flora do Brasil. Jardim Botânico do Rio de Janeiro. Available in: $<$ http://floradobrasil.jbrj.gov.br/jabot/floradobrasil/FB13181>. Access in: Jul 26th, 2018.

CARMO, F.F. \& JACOBI, C.M. 2013. A vegetação de canga no Quadrilátero Ferrífero, Minas Gerais: caracterização e contexto fitogeográfico. Rodriguésia, 64(3):527-541.

CARMO, F.F., CARMO, F.F., CAMPOS, I.C. \& JACOBI, C.M. 2012. Cangas: ilhas de ferro estratégicas para a conservação. Ciênc. Hoje 295:48-53.

CHUKR, N. 2015. Cipura. In Lista de Espécies da Flora do Brasil. Jardim Botânico do Rio de Janeiro. Available in: <http://floradobrasil.jbrj. gov.br/jabot/floradobrasil/FB30043>. Access in: Jul 26th, 2018.

COELHO, A.A.O.P. \& ZAPPI, D. 2015. Portulacaceae. In Lista de Espécies da Flora do Brasil. Jardim Botânico do Rio de Janeiro. Available in: <http://floradobrasil.jbrj.gov.br/jabot/floradobrasil/ FB20621>. Access in: Jul 26th, 2018.

CORTÊS, A.L.A. \& RAPINI, A. 2013. Justicieae (Acanthaceae) do Semiárido do Estado da Bahia, Brasil. Hoehnea 40(2): 253-292.

COSTA-E-SILVA, M.M. 2000. Cleome eosina J. F. Macbr. (Capparaceae Juss.), a new record for Brazil. Ernstia, 10(2):43-46.

CRIA. Centro de Referência e Informação Ambiental. 2018. SpeciesLink. Available in: http://www.splink.org.br/index. Access in: Jul 26th, 2018.

DAMASCENO-JUNIOR, G.A. 2005. Estudo florístico e fitossociológico de um gradiente altitudinal no Maciço do Urucum, Mato Grosso do Sul, Brasil. Tese de Doutorado, Instituto de Biologia, UNICAMP, Campinas.

DAMASCENO-JUNIOR, G.A., POTT, A., NEVES, D.R.M., SCIAMARELLI, A. \& FINA, B.G. 2018. Flora lenhosa de Florestas estacionais do estado de Mato Grosso do Sul: estado da arte. Iheringia, Sér. Bot., 73(supl.):65-79.

DEL'ARCO, J.O., SILVA, R.H., TARAPANOFF, I., FREIRE, F.A., PEREIRA, L.G.M., SOUZA, S.L., LUZ, D.S., PALMEIRA, R.C.B. \& TASSINARI, C.C.G. 1982. Geologia. In Projeto RADAMBRASIL Folha SE. 21 - Corumbá e parte da Folha SE.20 (Levantamento de Recursos Naturais, 27). Ministério de Minas e Energia, Rio de Janeiro, p.25-160.

DUTILH, J.H.A. \& OLIVEIRA, R.S. 2015. Amaryllidaceae. In Lista de Espécies da Flora do Brasil. Jardim Botânico do Rio de Janeiro. Available in: <http://floradobrasil.jbrj.gov.br/jabot/floradobrasil/ FB25116>. Access in: Jul 30th, 2018.

ESTEVES, G. \& TAKEUCHI, C. 2015. Gaya. In Lista de Espécies da Flora do Brasil. Jardim Botânico do Rio de Janeiro. Available in: <http://floradobrasil.jbrj.gov.br/jabot/floradobrasil/FB9062>. Access in: Jul 26th, 2018. 
ESTEVES, G. 2015. Waltheria. In Lista de Espécies da Flora do Brasil. Jardim Botânico do Rio de Janeiro. Available in: $<$ http://floradobrasil.jbrj.gov.br/jabot/floradobrasil/FB25773>. Access in: Jul 26th, 2018.

FARINACCIO, M.A. \& SIMÕES, A.O. 2018. Check-list das Apocynaceae do estado de Mato Grosso do Sul, Brasil. Iheringia, Sér. Bot., 73(supl.):131-146.

FIASCHI, P. 2018. Check-list da família Oxalidaceae no estado de Mato Grosso do Sul. Iheringia, Sér. Bot., 73(supl.):297-300.

FLORA DO BRASIL 2020 em construção. 2018. Jardim Botânico do Rio de Janeiro. Available in: <http://floradobrasil.jbrj.gov.br/>. Access in: Sep 4th, 2018.

FORZZA, R.C., BAUMGRATZ, J.F.A., BICUDO, C.E.M., CARVALHO-JR., A.A., COSTA, A., COSTA, D.P., HOPKINS, M.G., LEITMAN, P.M., LOHMANN, L.G., MAIA, L.C., MARTINELLI, G., MENEZES, M., MORIM, M.P., COELHO, M.A.N., PEIXOTO, A.L., PIRANI, J.R., PRADO, J., QUEIROZ, L.P., SOUZA, V.C., STEHMANN, J.R., SYLVESTRE, L.S., WALTER, B.M.T. \& ZAPPI, D. (ORG.). 2010. Catálogo de plantas e fungos do Brasil. Volumes 1 e 2. Andrea Jakobsson Estúdio, Instituto de Pesquisas Jardim Botânico do Rio de Janeiro, Rio de Janeiro. 1700p.

FRANCENER, A., ALMEIDA, R.F. \& SEBASTIANI, R. 2018. Check-list de Malpighiaceae do estado de Mato Grosso do Sul. Iheringia, Sér. Bot., 73(supl.):264-272.

GIULIETTI, A.M., RAPINI, A., ANDRADE, M.J.G., QUEIROZ, L.P. \& SILVA, J.M.C. 2009. Plantas Raras do Brasil. Conservation International, Belo Horizonte.

GROPPO, M., MARGALHO, L.F., FERREIRA, P.L. \& ERBERT, C. 2018. Check-list de Sapindaceae (Angiospermae) do estado de Mato Grosso do Sul, Brasil. Iheringia, Sér. Bot., 73(supl.):342-347.

GUGLIERI-CAPORAL, A., POTT, A., FELISMINO, M.F., CAPORAL, F.J.M. \& VALLS, J.F.M. 2018. Check-list das Poaceae do estado de Mato Grosso do Sul, Brasil. Iheringia, Sér. Bot., 73(supl.):313-328.

HARLEY, R., FRANÇA, F., SANTOS, E.P., SANTOS, J.S. \& PASTORE, J.F. 2015. Lamiaceae. In Lista de Espécies da Flora do Brasil. Jardim Botânico do Rio de Janeiro. Available in: $<$ http://floradobrasil.jbrj.gov.br/jabot/floradobrasil/FB59379>. Access in: Jul 26th, 2018.

IUCN. 2018. The IUCN Red List of Threatened Species, Version 2018-1, IUCN Red List Unit, Cambridge. Available in: <http://www. iucnredlist.org>. Access in: Sep 4th, 2018.

JABOT. 2018. Banco de Dados da Flora Brasileira. JBRJ - Instituto de Pesquisas Jardim Botânico do Rio de Janeiro. Available in: $<$ http://cor.jbrj.gov.br/v2/consulta.php > . Access in: Sep 5th, 2018.

JACOBI C.M. \& CARMO, F.F. 2011. Life-forms, pollination and seed dispersal syndromes in plant communities on ironstone outcrops, SE Brazil. Acta Bot. Bras. 25:395-412.

JACOBI, C.M. \& CARMO, F.F. 2008a. The contribution of ironstone outcrops to plant diversity in the Iron Quadrangle, a threatened Brazilian landscape. Ambio 37:324-326.

JACOBI, C.M. \& CARMO, F.F. 2008b. Diversidade dos campos rupestres ferruginosos no Quadrilátero Ferrífero, MG. Megadiversidade 4:24-32.

JACOBI, C.M., CARMO, F.F., CARMO, F.F. \& CAMPOS, I.C. 2015. Iron geosystems: priority areas for conservation in Brazil. In Mining in ecologically sensitive landscapes (M.Tibbett, ed.). Csiro Publishing. Leiden, p. 55-77.
JACOBI, C.M., CARMO, F.F., VINCENT, R.C. \& STEHMANN, J.R. 2007. Plant communities on the ironstone outcrops - a diverse and endangered Brazilian ecosystem. Biodivers. Conserv. 16:2185-2200.

JARDIM, A., KILLEEN, T.J. \& FUENTES, A. 2003. Guia de los árboles y arbustos del Bosque Seco Chiquitano, Bolivia. Editorial Fundación Amigos de La Naturaleza, Santa Cruz.

KOCH, I., RAPINI, A., SIMÕES, A.O., KINOSHITA, L.S., SPINA, A.P. \& CASTELLO, A.C.D. 2015. Apocynaceae. In Lista de Espécies da Flora do Brasil. Jardim Botânico do Rio de Janeiro. Available in: <http://floradobrasil.jbrj.gov.br/jabot/floradobrasil/ FB4601>. Access in: Jul 26th, 2018.

LINARES-PALOMINO, R., PENNINGTON, R.T. \& BRIDGEWATER, S. 2003. The phytogeography of the seasonally dry tropical forests in Equatorial Pacific South America. Candollea 58:473-499.

LOBÃO, A.Q., LOPES, J.C. \& MELLO-SILVA, R. 2018. Check-list das Annonaceae do estado de Mato Grosso do Sul, Brasil. Iheringia, Sér. Bot., 73(supl.):123-126.

LOIOLA, M.I.B. \& CORDEIRO, L.S. 2018. Check-list das Erythroxylaceae no estado de Mato Grosso do Sul, Brasil. Iheringia, Sér. Bot., 73(supl.):201-206.

LONGHI-WAGNER, H.M. 2015. Sporobolus. In Lista de Espécies da Flora do Brasil. Jardim Botânico do Rio de Janeiro. Available in: <http://floradobrasil.jbrj.gov.br/jabot/floradobrasil/FB13626>. Access in: Jul 26th, 2018.

LOUREIRO, R.L., LIMA, J.P.S. \& FONZAR, B.C. 1982. Vegetação. In Projeto RADAMBRASIL Folha SE. 21 - Corumbá e parte da Folha SE.20 (Levantamento de Recursos Naturais, 27). Ministério de Minas e Energia, Rio de Janeiro, p.329-372.

MACHATE, D.J., ALVES, F.M. \& FARINACCIO, M.A. 2016. Aspidosperma (Apocynaceae) no estado de Mato Grosso do Sul, Brasil. Rodriguésia 67(4):1011-1024.

MARTINELLI, G. \& MORAES, M.A. (orgs.) 2013. Livro Vermelho da Flora do Brasil. Centro Nacional de Conservacao da Flora (CNCFLORA), Rio de Janeiro.

MESSIAS, M.C.T.B., LEITE, M.G.P., MEIRA-NETO, J.A.A. \& KOZOVITS, A.R. 2012. Fitossociologia de campos rupestres quartzíticos e ferruginosos no Quadrilátero Ferrífero, Minas Gerais. Acta Bot. Bras. 26(1): 230-242.

MOBOT. Missouri Botanical Garden. 2018. Tropicos.org. Missouri Botanical Garden. Available in: http://www.tropicos.org/ Name/8500374. Access in: Jul 26th, 2018.

MOTA, N.F.O., SILVA, L.V.C., MARTINS, F.D. \& VIANA, P.L. 2015. Vegetação sobre sistemas ferruginosos da Serra dos Carajás. In Geossistemas Ferruginosos do Brasil: áreas prioritárias para conservação da diversidade geológica e biológica, patrimônio cultural e serviços ambientais (F.F. do Carmo \& L.H.Y. Kamino, eds.), 3i Editora, Belo Horizonte, p. 289-315.

MOURÃO, A. \& STEHMANN, J.R. 2007. Levantamento da flora do campo rupestre sobre canga hematítica couraçada remanescente na Mina do Brucutu, Barão de Cocais, Minas Gerais, Brasil. Rodriguésia 58:775-786.

OLIVEIRA, P.P. 2016. A influência do tamanho insular sobre a fenologia de plantas em bancadas lateríticas (cangas) de Corumbá, Mato Grosso do Sul. Dissertação de Mestrado, Universidade Federal de Mato Grosso do Sul, Campo Grande. 
PAGGI, G.M., LOUZADA, R.B., ISHII, I.H., TAKAHASI, A., ARRUDA, R.C.O. \& LORENZ-LEMKE, A.P. 2015. Rediscovering Dyckia excelsa (Bromeliaceae) in Mato Grosso do Sul, Brazil: taxonomy, geographic distribution and notes on leaf anatomy. Systematic Botany 40(1):129-135.

PANFIGLIO, T., CORNEJO, X. \& FARINACCIO, M.A. 2018. Check-list de Capparaceae do estado de Mato Grosso do Sul, Brasil. Iheringia, Sér. Bot., 73(supl.):174-177.

PASTORE, J.F., LUDTKE, R., FERREIRA, D.M.C. \& KUNTZ, J. 2015. Polygalaceae. In Lista de Espécies da Flora do Brasil. Jardim Botânico do Rio de Janeiro. Available in: < http://floradobrasil.jbrj. gov.br/jabot/floradobrasil/FB127266>. Access in: Jul 26th, 2018.

PELLEGRINI, M.O.O., FORZZA, R.C. \& SAKURAGUI, C.M. 2017. Novelties in Brazilian Tradescantia L.(Commelinaceae). PhytoKeys 80: $1-31$

PIFANO, D.S., VALENTE, A.S.M., ALMEIDA, H.S., MELO, P.H.A., CASTRO, R.M. \& VAN DEN BERG, E. 2010. Caracterização florística e fitofisionômica da Serra do Condado, Minas Gerais, Brasil. Biota Neotropica, 10(1): http://www.biotaneotropica.org.br/ v10n1/pt/fullpaper?bn01010012010+pt. Last acess: Aug 5th, 2018.

POTT, A. \& POTT, V.J. 1996. Flora do Pantanal - listagem atual de fanerógamas. In Anais do Simpósio sobre Recursos Naturais e Sócio-Econômicos do Pantanal, Manejo e Conservação, 2, Embrapa Pantanal, Corumbá, p.297-325.

POTT, A. \& POTT, V.J. 2003. Espécies de fragmentos florestais em Mato Grosso do Sul. In Fragmentação florestal e alternativas de desenvolvimento rural na região Centro-Oeste (R.B. Costa, ed.). UCDB, Campo Grande, p.26-52.

PRADO, D.E. \& GIBBS, P. 1993. Patterns of species distributions in the dry seasonal forests of South America. Ann. Missouri Bot. Gard. 80(4):902-927.

PROENÇA, C.E.B., SOARES-SILVA, L.H., VILLARROEL, D., GOMES-BEZERRA, K.M., ROSA, P.O., FARIA, J.E.Q. \& SOBRAL, M. 2018. Flora do Mato Grosso do Sul: Myrtaceae. Iheringia, Sér. Bot., 73(supl.):277-282.

PROFICE, S.R., KAMEYAMA, C., CÔRTES, A.L.A., BRAZ, D.M., INDRIUNAS, A., VILAR, T., PESSOA, C., EZCURRA, C. \& WASSHAUSEN, D. 2015. Acanthaceae. In Lista de Espécies da Flora do Brasil. Jardim Botânico do Rio de Janeiro. Available in: $<$ http://floradobrasil. jbrj.gov.br/jabot/floradobrasil/FB104453>. Access in: Jul 26th, 2018.

RIZZINI, C.T. 1997. Tratado de fitogeografia do Brasil: aspectos ecológicos, sociológicos e florísticos. 2 ed. Âmbito Cultural Edições, Rio de Janeiro.

ROQUE, N., TELES, A.M., MOURA, L., PACHECO, R.A., SILVA, G.H.L., ALVES, M. \& NAKAJIMA, J.N. 2018. Check-list de Asteraceae no estado de Mato Grosso do Sul, Brasil. Iheringia, Sér. Bot., 73(supl.):147-156.

SARTORI, A.L.B., LIMA, L.C.P., POTT, V.J., VALLS, J.F.M., CRISTALDO, A.C.M., POLIDO, C.A., COSTA, L.C., POTT, A., FORTUNA-PEREZ, A.P., SILVA, G.M., VAZ, A.M.S.F., BORTOLUZZI, R..L.C., PESTANA, L.T.C., SILVA, R.R., SOUZALIMA, E.S., MANSANO, V.F. \& SCIAMARELLI, A. Check-list das Leguminosae do estado de Mato Grosso do Sul. Iheringia, Sér. Bot., 73(supl.):239-254.
SCHÜTZ, N. 2014. Deuterocohnia meziana (Bromeliaceae): Subspecies classification and the description of the new subspecies D. meziana subsp. vogtii from northern Paraguay. Phytotaxa 162(1):18-30.

SECCO, R.S., BIGIO, N.C., CORDEIRO, I. PSCHEIDT, A..C. MARQUES O. \& CARUZO, M.B.R. 2018. Check-list de Euphorbiaceae s. str., Phyllanthaceae e Peraceae de Mato Grosso do Sul, Brasil. Iheringia, Sér. Bot., 73(supl.):207-215.

SENNA, L. 2015. Alternanthera. In Lista de Espécies da Flora do Brasil. Jardim Botânico do Rio de Janeiro. Available in: $<$ http://floradobrasil.jbrj.gov.br/jabot/floradobrasil/FB139767>. Access in: Jul 26th, 2018.

SILVA, J.S.V., POTT, A., CARDOSO, E. L., MORAES, A. S., SALIS, S. M., POTT, V. J., MAURO, R. de A. \& GALDINO, S. G. 2000. Avaliação integrada do Maciço do Urucum e adjacências procedimentos e diretrizes. In Zoneamento ambiental da borda oeste do Pantanal: Maciço do Urucum e adjacências (J.S.V.SILVA, ed.). Embrapa Informação e Tecnologia, Brasília, p.9-22.

SIMÃO-BIANCHINI, R. \& FERREIRA, P.P.A. 2015. Evolvulus. In Lista de Espécies da Flora do Brasil. Jardim Botânico do Rio de Janeiro. Available in: <http://floradobrasil.jbrj.gov.br/jabot/ floradobrasil/FB6994>. Access in: Jul 26th, 2018.

SKIRYCZ A., CASTILHO A., CHAPARRO C., CARVALHO N., TZOTZOS G. \& SIQUEIRA J.O. 2014. Canga biodiversity, a matter of mining. Front. Plant Sci. 5:653.

SOARES-NETO, R.L., THOMAS, W.W., BARBOSA, M.R.V. \& ROALSON, E.H. 2018. New combinations and taxonomic notes for Tarenaya (Cleomaceae). Acta Bot. Bras. Epub March 19. Available in: <https://dx.doi.org/10.1590/0102-33062017abb0417>.

SOBRAL, M., PROENÇA, C., SOUZA, M., MAZINE, F. \& LUCAS, E. 2015. Myrtaceae. In Lista de Espécies da Flora do Brasil. Jardim Botânico do Rio de Janeiro. Available in: < http://floradobrasil.jbrj. gov.br/jabot/floradobrasil/FB37110>. Access in: Jul 26th, 2018.

SORIANO, B.M. 1997. Caracterização Climática de Corumbá-MS. Boletim de Pesquisa, 11, Embrapa, Corumbá.

SOUZA, C. R. \& CARMO, F. F. 2015. Geossistemas ferruginosos no Brasil. In Geossistemas Ferruginosos do Brasil (F.F. do Carmo \& L.H.Y. Kamino, eds.), 3i Editora, Belo Horizonte, p.47-76.

SOUZA, V.C. \& BORTOLUZZI, R.L.C. 2015. Chamaecrista. In Lista de Espécies da Flora do Brasil. Jardim Botânico do Rio de Janeiro. Available in: <http://floradobrasil.jbrj.gov.br/jabot/floradobrasil/ FB82933>. Access in: Jul 26th, 2018.

TAKAHASI, A. \& MEIRELLES, S.T. 2014 Ecologia da vegetação herbácea de bancadas lateríticas (cangas) em Corumbá, MS, Brasil. Hoehnea 41(4):515-528.

TAKAHASI, A. 2010. Ecologia da vegetação em bancadas lateríticas em Corumbá, MS. Tese de doutorado, Universidade de São Paulo, São Paulo.

TAKAHASI, A. 2015. Flora das cangas de Corumbá, MS: Diversidade e conservação. In Geossistemas Ferruginosos do Brasil (F.F. do Carmo \& L.H.Y. Kamino, eds.), 3 i Editora, Belo Horizonte, p.317-334.

TIBBETT, M. 2015. Mining in ecologically sensitive landscapes: concepts and challenges. In Mining in ecologically sensitive landscapes (M.Tibbett, ed.). Csiro Publishing. Leiden, p.3-6. 
TOMÁS, W.M., ISHII, I.H., STRUSSMANN, C., NUNES, A.P., SALIS, S.M., CAMPOS, Z., FERREIRA, V.L., BORDIGNON, M.O., BARROS, A.T.M. \& PADILHA, D.R.C. 2010. Borda oeste do Pantanal e Maciço do Urucum em Corumbá, MS: área prioritária para conservação da biodiversidade. In Anais do V Simpósio sobre Recursos Naturais e Socioeconômicos do Pantanal (W.M.Tomás, coord), Embrapa Pantanal, Corumbá, p.1-6.

VERSIEUX, L.M., COFFANI-NUNES, J.V., PAGGI, G.M. \& COSTA, A.F. 2018. Check-list of Bromeliaceae from Mato Grosso do Sul, Brazil. Iheringia, Sér. Bot., 73(supl.):163-168.
VIANA, P.L. \& LOMBARDI, J.A. 2007. Florística e caracterização dos campos rupestres sobre canga na Serra da Calçada, Minas Gerais, Brasil. Rodriguésia 58(1): 159-177.

ZAPPI, D.C., TAYLOR, N.P., DAMASCENO-JUNIOR, G.A., POTT, V.J. \& MACHADO, M.C. 2018. Check-list das Cactaceae do estado do Mato Grosso do Sul, Brasil. Iheringia, Sér. Bot., 73(supl.):169173.

Received: $29 / 01 / 2019$

Revised: 22/05/2019

Accepted: 27/05/2019

Published online: 01/07/2019 